\title{
Bifurcation and dynamic response analysis of a rotating blade excited by upstream vortices*
}

\author{
Dan Wang ${ }^{1}, \quad$ Yushu Chen ${ }^{1}, \quad$ Marian Wiercigroch ${ }^{2}, \quad$ Qingjie Cao ${ }^{1}$ \\ (1. School of Astronautics, Harbin Institute of Technology, Harbin 150001, China; \\ 2. Centre for Applied Dynamics Research, School of Engineering, \\ University of Aberdeen, King's College, Aberdeen AB24 3UE, Scotland, UK)
}

\begin{abstract}
A reduced model simulating vortex-induced vibrations (VIVs) for turbine blades is proposed and analyzed. The rotating blade is modeled as a uniform cantilever beam while a van der Pol oscillator is used to represent the time-varying characteristics of the vortex shedding, which interacts with equations of motion for a blade to simulate the fluid-structure interaction. The action for the structural motion on the fluid is considered as a linear inertia coupling. Nonlinear characteristics for the dynamic responses are investigated with the multiple scale method and the modulation equations are derived. The transition set consisting of the bifurcation and hysteresis sets is constructed by using the singularity theory and the effects of system parameters including the van der Pol damping and the coupling parameter on the equilibrium solutions are analyzed. Frequency-response curves are obtained and the stabilities are determined by using the Routh-Hurwitz criterion. The phenomena including the saddle-node and Hopf bifurcations are found to occur under certain parameter values. A direct numerical method is used to analyze the dynamic characteristics for the original system and verify the validity of the multiple scale method. The results indicate that the new coupled model can be useful to explain the rich dynamic response characteristics including possible bifurcation phenomena in the VIVs.
\end{abstract}

Key words vortex-induced vibration, van der Pol oscillator, dynamic responses, transition set, singularity theory, bifurcation phenomenon

Chinese Library Classification O322

2010 Mathematics Subject Classification 70K30, 70K50

\section{Introduction}

The phenomenon of vortex shedding is often observed in engineering, such as circular cylinders in cross-flow ${ }^{[1]}$, oscillating airfoils and turbomachine blades ${ }^{[2]}$ as well as micro air vehicles ${ }^{[3]}$. Different vortex patterns shedding from the trailing edge of a NACA 0012 airfoil that was oscillated sinusoidally in plunge were captured in water tunnel tests with a Reynolds number range based on the airfoil chord from 500 to $21,000^{[2]}$. A study showed that a strong similarity between the vortex wakes shed from cylinders and airfoils with the sinusoidal plunge motion in the low-speed flow and the wakes shed from a turbine nozzle cascade in transonic flow ${ }^{[4]}$.

* Received ; / Revised ;

Project supported by the National Basic Research Program of China (973 Project)

(No. 2015CB057405) and the National Natural Science Foundation of China (No. 11372082)

Corresponding author: Dan Wang, Ph.D, E-mail: danwang2014518@hotmail.com 
The thermo-acoustic effect associated with the vortex shedding was presented in the rotating machines ${ }^{[4]}$. A turbine blade with a blunt trailing edge was found to shed a von Kármán vortex street ${ }^{[5,6]}$, which can result in a depression for the base pressure and separation for the energy. The aeroelastic problems could occur easily in operating conditions owing to the vortex shedding, such as the flutter for the aircraft wings, helicopter as well as turbine blades.

As a fluid-structure interaction (FSI) problem, the vortex-induced vibration is difficult in modeling the coupled loads. Because both experimental ${ }^{[7]}$ and numerical ${ }^{\left[{ }^{-11}\right.}{ }^{-1}$ approaches are expensive to obtain robust results, simplified models have been developed to reveal nonlinear effects of system parameters on the dynamic responses of the system. Generally turbine blades are simplified as the elastic clamped-free beams both in experimental ${ }^{[12]}$ and numerical analysis [13-16]. The two-dimensional friction contact problem and impact vibration characteristics of a rotational blade in the centrifugal force field were analyzed ${ }^{[17,18]}$, in which the blade was simplified as a continuous cantilever.

There is a great number of studies about the van der Pol model for the FSI problem, which can lead to the self-excited oscillation for the structure owing to the characteristic of time-varying ${ }^{[19-21]}$. Specifically, a van der Pol-based model was introduced ${ }^{[23]}$, which captured many of the features seen in experimental results and has been subsequently modified and improved ${ }^{[24]}$. A van der Pol oscillation was used to model an aeroelastic system possessing limit cycle oscillations ${ }^{[22]}$. The vortex-induced vibrations for a cylinder ${ }^{[25]}$, an offshore riser ${ }^{[26]}$ and a turbine blade ${ }^{[27]}$ were analyzed, where the time-varying characteristic of the vortices was modeled by the van der Pol oscillation and effects of the structural motion on the fluid were also studied. A van der Pol damping term was added to the equations of four coupled elastic beams to represent the self-excitation and fluid-structure interaction of the system ${ }^{[15]}$. The FSI problem for turbine blades was simulated by adding a van der Pol self-exciting term to a single partial differential equation of a linear beam ${ }^{[16]}$. The van der Pol oscillator was used to simulate the time-varying characteristics of the lift coefficient for the fluid-structure interactions of turbine blades ${ }^{[28]}$.

In addition, there is a great body of research demonstrated that the vortex shedding from oscillating airfoils and cylinders was significantly affected by the body oscillation ${ }^{[1,25,26,29-33]}$. Hence in order to analyze the vibrations of the vortices further, it is necessary to consider the action of the structural motion on the fluid.

The motivation of this paper is to investigate the dynamic response and bifurcation characteristics for vortex-induced vibrations of a rotating blades. The blade is modeled as a cantilever beam while a van der Pol equation is used to mimic the time-varying characteristics for the vortices. The reaction of the structural motion on the fluid is considered and represented by a linear inertial coupling. The 1:1 internal resonance analysis is carried out with the multiple scale method. The two-parameter bifurcation diagram is derived by using the singularity theory and frequency-response curves in different parameter regions are obtained. The time histories, phase portrait and Lyapunov exponents are computed by the Runge-Kutta method for the original system to verify the validity of the multiple scale method.

\section{Mathematical modelling}

\subsection{The cantilever beam model for the blade}

In engineering practice, the rotating Euler-Bernoulli beam ${ }^{[34,35]}$ is often used to analyze the dynamic characteristics of engineering systems, such as the turbomachinery, wind turbines, robotic manipulators and rotorcraft blades. In order to investigate the interaction mechanism of the structure and fluid, the blade is assumed as a continuous uniform straight cantilever beam based on the Euler-Bernoulli formulation in the centrifugal force field as shown in Fig.1.

The transverse motion of the beam would be mainly studied and the effects of shear deformation and rotatory inertia are neglected. In addition, the study in [35] about the in plane 
(a)

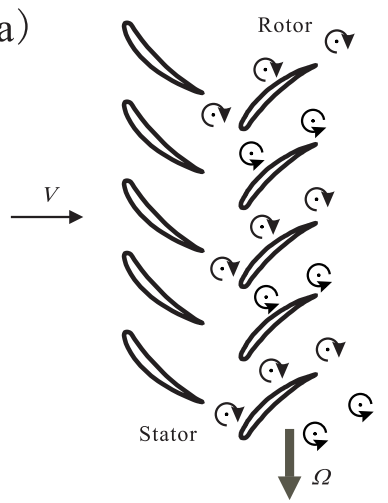

(b)

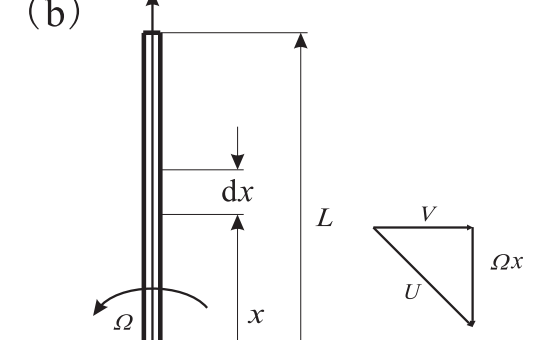

Fig. 1 Sketches of the blades excited by the upstream vortices: (a) The axial cascade; (b) a cantilever beam model.

vibrations of a rotating Euler-Bernoulli beam with considering the Coriolis force revealed that all the coefficients that were related to the Coriolis force vanish during the reduction processing, where a single degree-of-freedom model for the $i$-th mode vibration was extracted by using the Galerkin method. Hence, there is no Coriolis term in the single degree-of-freedom equation. Moreover, the study of Xu et al. ${ }^{[36]}$ demonstrated that the Coriolis force has no influence on the axial and torsional vibrations of the rotating blades, and the effect on tangential vibration is very small and can be neglected. Therefore, the effect of the Coriolis force on the rotating blade is neglected in this study.

The transverse displacement of the cantilever beam is assumed as $w(x, t)$, and the equation of the blade motion can be derived by considering the equilibrium of forces and moments acting on the differential segment of the blade with the length of $\mathrm{d} x$ as shown in Fig.2, please refer to detailed analysis in the article [17].

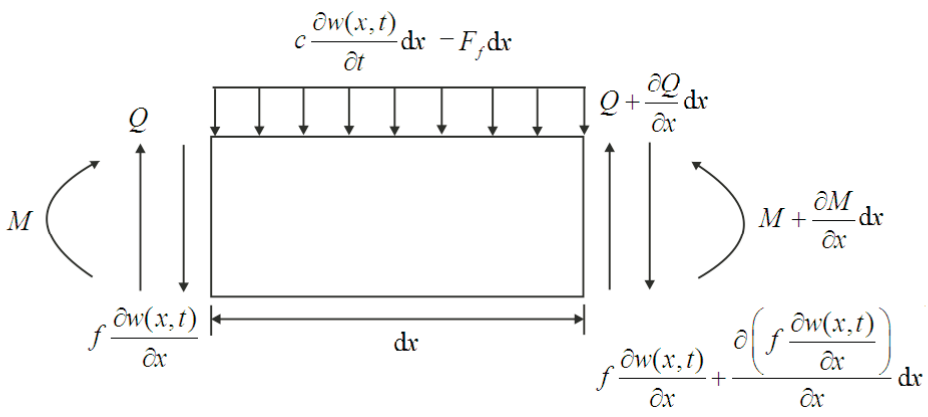

Fig. 2 Free-body diagram of the differential segment.

The first dynamic equilibrium relationship with respect to the transverse displacement $w(x, t)$ can be obtained by summing all the forces in the transverse direction for the segment,

$$
\begin{array}{r}
Q-f \frac{\partial w(x, t)}{\partial x}-\left[Q+\frac{\partial Q}{\partial x} \mathrm{~d} x-f \frac{\partial w(x, t)}{\partial x}-\frac{\partial}{\partial x}\left(f \frac{\partial w(x, t)}{\partial x}\right) \mathrm{d} x\right]- \\
c \frac{\partial w(x, t)}{\partial t} \mathrm{~d} x+F_{f} \mathrm{~d} x=m \mathrm{~d} x \frac{\partial^{2} w(x, t)}{\partial t^{2}},
\end{array}
$$

where $m=\left(\rho+\rho_{f}\right) A$ is the total mass consisting of the structure and the added mass induced by 
the fluid, $\rho$ and $\rho_{f}$ are the densities of the structure and air flow, respectively, $A$ is the area of the cross-section for the cantilever beam. $\rho$ and $A$ are constants according to the beam assumption. $Q$ is the shear force acting on the cross-section of the blade, $c$ is the viscous damping coefficient. $f$ is the axial load expressed as $f(x)=\int_{x}^{L} \rho A \Omega^{2} \xi \mathrm{d} \xi=\frac{1}{2} \rho A \Omega^{2}\left(L^{2}-x^{2}\right)$ as shown in Fig. 1(b), $L$ is the length of the blade, $\Omega$ is the rotating speed of the blade. $F_{f}$ is the fluid force acting on the blade induced by the vortices which reads $F_{f}=\frac{1}{2} \rho_{f} U^{2} D_{0} C_{L}, C_{L}$ is the lift coefficient which represents the time-varying characteristics of the vortices, $U=\sqrt{V^{2}+(\Omega x)^{2}}$ is the total velocity, $V$ is the freestream velocity, $D_{0}$ is the characteristic length of the cross-section of the cantilever beam.

Neglecting the shear deformation and the rotation of the cross-section according to the beam assumption, the basic moment-curvature relationship is introduced when the condition of moment equilibrium is satisfied, which reads

$$
Q=\frac{\partial M}{\partial x}=\frac{\partial}{\partial x}\left(E I \frac{\partial^{2} w(x, t)}{\partial x^{2}}\right)
$$

where $M$ is the moment acting on the cross-section of the beam, and $E I$ is the flexural rigidity of the structure. Combining with Eq. (2), Eq. (1) can be simplified as

$$
E I \frac{\partial^{4} w(x, t)}{\partial x^{4}}+m \frac{\partial^{2} w(x, t)}{\partial t^{2}}+c \frac{\partial w(x, t)}{\partial t}=F_{f}-\rho A \Omega^{2} x \frac{\partial w(x, t)}{\partial x}+\frac{1}{2} \rho A \Omega^{2}\left(L^{2}-x^{2}\right) \frac{\partial^{2} w(x, t)}{\partial x^{2}} .
$$

With non-dimensional variables $v(x, t)=\frac{w(x, t)}{D_{0}}, z=\frac{x}{L}, \tau=\Omega t, \omega_{0}=\sqrt{\frac{E I}{m L^{4}}}, \zeta=\frac{c}{m \Omega}$ into Eq. (3), yields

$$
\frac{\omega_{0}^{2}}{\Omega^{2}} \frac{\partial^{4} v(z, \tau)}{\partial z^{4}}+\frac{\partial^{2} v(z, \tau)}{\partial \tau^{2}}+\zeta \frac{\partial v(z, \tau)}{\partial \tau}=\frac{F_{f}}{m D_{0} \Omega^{2}}-\frac{\rho A z}{m} \frac{\partial v(z, \tau)}{\partial z}+\frac{\rho A\left(1-z^{2}\right)}{2 m} \frac{\partial^{2} v(z, \tau)}{\partial z^{2}} .
$$

The boundary conditions of the cantilever beam should meet the following conditions:

for $z=0$, the displacement and the rotation of the beam should be equal to zero, that is,

$$
v(0, \tau)=0, v^{\prime}(0, \tau)=0,
$$

for $z=1$, the moment and the shear force should be equal to zero, that is,

$$
v^{\prime \prime}(1, \tau)=0, v^{\prime \prime \prime}(1, \tau)=0 .
$$

\subsection{The van der Pol oscillator}

The van der Pol oscillator is often used to model the time-varying and self-sustained characteristics of the flow in the analytical/experimental research of vortex-induced vibrations. The lift coefficient as a physical variable can represent the flow characteristics ${ }^{[25,26]}$, which can affect the lift force and then the structural vibrations. Herein, the van der Pol oscillator is introduced to simulate the time-varying characteristics of the lift coefficient, that is

$$
\frac{\partial^{2} q(z, t)}{\partial t^{2}}+\lambda \omega_{f}\left[q^{2}(z, t)-1\right] \frac{\partial q(z, t)}{\partial t}+\omega_{f}^{2} q(z, t)=F_{s}
$$

where $q(z, t)=2 C_{L} / C_{L 0}, C_{L 0}$ is the reference lift coefficient, $\omega_{f}=2 \pi \mathrm{St} V / D_{0}$ is the shedding frequency of the vortex, St is the Strouhal number, $\lambda$ is the van der Pol damping coefficient. In addition, the action of the structural vibration on the fluid motion is considered as outlined in the Introduction, denoting by $F_{s}$. The study in $[25,26]$ showed that, the inertial coupling was 
the ideal form to describe the reaction of blades to the flows. So the linear inertial coupling is studied and the force $F_{s}$ induced by the structural motion can be assumed as $F_{s}=\tilde{N} \frac{\partial^{2} v(z, t)}{\partial t^{2}}$, $\tilde{N}$ is the linear coupling parameter.

To analyze in the same time scale, letting $\tau=\Omega t$, Eq. (5) becomes

$$
\frac{\partial^{2} q(z, \tau)}{\partial \tau^{2}}+\lambda\left(\frac{\omega_{f}}{\Omega}\right)\left[q^{2}(z, \tau)-1\right] \frac{\partial q(z, \tau)}{\partial \tau}+\left(\frac{\omega_{f}}{\Omega}\right)^{2} q(z, \tau)=\tilde{N} \frac{\partial^{2} v(z, \tau)}{\partial \tau^{2}} .
$$

Hence, the coupled Eqs (4) and (6) model the interactions of the blade and vortices.

\subsection{The reduced model with Galerkin discretization}

An arbitrary oscillation of the structure $v(z, \tau)$ can be accurately described with a sum of all of its modal responses, which is mathematically expressed with a Taylor series composed of individual modal components ${ }^{[37]}$ as follows,

$$
v(z, \tau)=\sum_{i=1}^{\infty} v_{i}(\tau) \tilde{v}_{i}(z)
$$

where $\tilde{v}_{i}(z)$ and $v_{i}(\tau)(i=1,2, \ldots)$ represent the mode-shapes and modal coordinates of the beam, respectively. When the shapes of the deformation are known, the modal coordinates $v_{i}(\tau)(i=1,2, \ldots)$ define how the amplitudes of the associated deformations $\tilde{v}_{i}(z)(i=1,2, \ldots)$ change with time. Thus the continuous system can be discretized by defining it on the modal subspaces.

For the approximate solution, the orthonormal sets of eigen-amplitude-functions for a cantilever beam as the modal functions for the blade are introduced, that is

$$
\tilde{v}_{i}(z)=\cosh \left(\beta_{i} z\right)-\cos \left(\beta_{i} z\right)-\frac{\cosh \beta_{i}+\cos \beta_{i}}{\sinh \beta_{i}+\sin \beta_{i}}\left[\sinh \left(\beta_{i} z\right)-\sin \left(\beta_{i} z\right)\right]
$$

where $\beta_{i}(i=1,2, \ldots)$ are the roots of the transcendental equation $\cos \beta \cosh \beta+1=0$ derived from the boundary conditions of the cantilever beam.

Herein, the cross-section of the beam is assumed as uniform, so the orthogonality of different modal functions can be written as

$$
\int_{0}^{1} \tilde{v}_{i}(z) \tilde{v}_{j}(z) \mathrm{d} z=0,(i \neq j) .
$$

It means that the spatial distributions of the structure are perpendicular to each other in modal space if they are not of the same order.

When the modal functions are in the same order, the integral can be calculated as

$$
\begin{aligned}
& \int_{0}^{1} \tilde{v}_{i}^{2}(z) \mathrm{d} z \\
& =\int_{0}^{1}\left\{\cosh \left(\beta_{i} z\right)-\cos \left(\beta_{i} z\right)-\frac{\cosh \beta_{i}+\cos \beta_{i}}{\sinh \beta_{i}+\sin \beta_{i}}\left[\sinh \left(\beta_{i} z\right)-\sin \left(\beta_{i} z\right)\right]\right\}^{2} \mathrm{~d} z \\
& =\frac{1}{2 \beta_{i}\left(\sin \beta_{i}+\sinh \beta_{i}\right)^{2}}\left\{\begin{array}{l}
6\left(1+\cos \beta_{i} \cosh \beta_{i}\right)\left(\cos \beta_{i} \sinh \beta_{i}-\sin \beta_{i} \cosh \beta_{i}\right) \\
+\beta_{i}\left[\cosh \left(2 \beta_{i}\right)+4 \sin \beta_{i} \sinh \beta_{i}\right]-\beta_{i} \cos \left(2 \beta_{i}\right)
\end{array}\right\} .
\end{aligned}
$$

It can be seen from Eq. (10) that the value of the integral would be determined by the corresponding coefficient $\beta_{i}$.

On the other hand, the findings of Skop and Balasubramanian ${ }^{[38]}$ as well as some other research about the span-wise distribution of the shedding variable were considered ${ }^{[39-43]}$, in which the spatial component of $q(z, \tau)$ is described with the shape of the respective normal 
mode and separated from the temporal part of the response. Hence, the form of the solution $q(z, \tau)$ can be assumed to be expressed with a Taylor series of modal components as analyzed in [39-43], that is

$$
q(z, \tau)=\sum_{i=1}^{\infty} q_{i}(\tau) \tilde{q}_{i}(z)
$$

where the relations between the mode-shapes $\tilde{q}_{i}(z)(i=1,2, \ldots)$ and the modal coordinates $q_{i}(\tau)(i=1,2, \ldots)$ are same as that of in Eq. $(7)$.

The modal functions for the van der Pol oscillator are introduced according to the study in [43], that is

$$
\tilde{q}_{i}(z)=\sin (i \pi z),(i=1,2, \ldots)
$$

which satisfy the orthogonality condition:

$$
\int_{0}^{1} \tilde{q}_{i}(z) \tilde{q}_{j}(z) \mathrm{d} z= \begin{cases}\frac{1}{2}, & i=j \\ 0, & i \neq j\end{cases}
$$

Substituting (7) into Eq. (4) and orthogonalizing the latter with respect to the set $\tilde{v}_{j}(z)$ and with the relation $\frac{\partial^{4} \tilde{v}_{i}(z)}{\partial z^{4}}=\beta_{i}^{4} \tilde{v}_{i}(z)$, one can obtain that

$$
\begin{array}{r}
\frac{\omega_{0}^{2}}{\Omega^{2}} \sum_{i=1}^{\infty} v_{i}(\tau) \beta_{i}^{4} \int_{0}^{1} \tilde{v}_{i}(z) \tilde{v}_{j}(z) \mathrm{d} z+\sum_{i=1}^{\infty} \frac{\partial^{2} v_{i}(\tau)}{\partial \tau^{2}} \int_{0}^{1} \tilde{v}_{i}(z) \tilde{v}_{j}(z) \mathrm{d} z+ \\
\zeta \sum_{i=1}^{\infty} \frac{\partial v_{i}(\tau)}{\partial \tau} \int_{0}^{1} \tilde{v}_{i}(z) \tilde{v}_{j}(z) \mathrm{d} z=\frac{C_{L 0} \rho_{f} L^{2}}{4 m} \sum_{i=1}^{\infty} q_{i}(\tau) \int_{0}^{1}\left[V^{2} /(L \Omega)^{2}+z^{2}\right] \tilde{q}_{i}(z) \tilde{v}_{j}(z) \mathrm{d} z- \\
\frac{\rho A}{m} \sum_{i=1}^{\infty} v_{i}(\tau) \int_{0}^{1} \frac{\partial \tilde{v}_{i}(z)}{\partial z} \tilde{v}_{j}(z) z \mathrm{~d} z+\frac{\rho A}{2 m} \sum_{i=1}^{\infty} v_{i}(\tau) \int_{0}^{1} \frac{\partial^{2} \tilde{v}_{i}(z)}{\partial z^{2}} \tilde{v}_{j}(z)\left(1-z^{2}\right) \mathrm{d} z .
\end{array}
$$

Because the fluid-structure interaction problem considered in this study is an ideal one, the fluid force can induce the structural oscillation in only one mode, so the van der Pol oscillator can also be expected to have the same and single modal distribution in the space. Hence, the first modal approximations of the cantilever beam and the van der Pol oscillator are investigated. is

The first mode equation of the structure can be derived from Eq. (14) when $i=j=1$, that

$$
\begin{array}{r}
\frac{\omega_{0}^{2}}{\Omega^{2}} v_{1}(\tau) \beta_{1}^{4} \int_{0}^{1} \tilde{v}_{1}^{2}(z) \mathrm{d} z+\frac{\partial^{2} v_{1}(\tau)}{\partial \tau^{2}} \int_{0}^{1} \tilde{v}_{1}^{2}(z) \mathrm{d} z+\zeta \frac{\partial v_{1}(\tau)}{\partial \tau} \int_{0}^{1} \tilde{v}_{1}^{2}(z) \mathrm{d} z= \\
\frac{C_{L 0} \rho_{f} L^{2}}{4 m} q_{1}(\tau) \int_{0}^{1}\left[V^{2} /(L \Omega)^{2}+z^{2}\right] \tilde{q}_{1}(z) \tilde{v}_{1}(z) \mathrm{d} z- \\
\frac{\rho A}{m} v_{1}(\tau) \int_{0}^{1} \frac{\partial \tilde{v}_{1}(z)}{\partial z} \tilde{v}_{1}(z) z \mathrm{~d} z+\frac{\rho A}{2 m} v_{1}(\tau) \int_{0}^{1} \frac{\partial^{2} \tilde{v}_{1}(z)}{\partial z^{2}} \tilde{v}_{1}(z)\left(1-z^{2}\right) \mathrm{d} z
\end{array}
$$

Thus first-order equation for the cantilever beam can be simplified as

$$
\frac{\mathrm{d}^{2} v_{1}(\tau)}{\mathrm{d} \tau^{2}}+\zeta \frac{\mathrm{d} v_{1}(\tau)}{\mathrm{d} \tau}+\omega_{s}^{2} v_{1}(\tau)=\left(b \omega_{R}^{2}+d\right) q_{1}(\tau)
$$

where $\omega_{R}=\omega_{f} / \Omega$ is the non-dimensional frequency of the fluid, $\omega_{s}=\sqrt{\bar{\omega}^{2}+a}$ is the non- 
dimensional frequency of the structure and $\bar{\omega}=\frac{\omega_{0}}{\Omega} \beta_{1}^{2}$,

$$
\begin{gathered}
a=\frac{\rho \mathrm{A}}{2 \mathrm{~m}} \frac{2 \int_{0}^{1} \frac{\partial \tilde{v}_{1}(z)}{\partial z} \tilde{v}_{1}(z) z \mathrm{~d} z-\int_{0}^{1} \frac{\partial^{2} \tilde{v}_{1}(z)}{\partial z^{2}} \tilde{v}_{1}(z)\left(1-z^{2}\right) \mathrm{d} z}{\int_{0}^{1} \tilde{v}_{1}^{2}(z) \mathrm{d} z}, \\
b=\frac{\rho_{f} C_{L 0} D_{0}^{2}}{16 m \pi^{2} \mathrm{St}^{2}} \frac{\int_{0}^{1} \tilde{q}_{1}(z) \tilde{v}_{1}(z) \mathrm{d} z}{\int_{0}^{1} \tilde{v}_{1}^{2}(z) \mathrm{d} z}, d=\frac{\rho_{f} L^{2} C_{L 0}}{4 m} \frac{\int_{0}^{1} \tilde{q}_{1}(z) \tilde{v}_{1}(z) z^{2} \mathrm{~d} z}{\int_{0}^{1} \tilde{v}_{1}^{2}(z) \mathrm{d} z} .
\end{gathered}
$$

The values of the above definite integrals can be obtained when the coefficient $\beta_{1}$ is determined.

Similarly, substituting (11) into Eq. (6) and orthogonalizing the latter with respect to the set $\tilde{q}_{j}(z)$, one can obtain that

$$
\begin{array}{r}
\sum_{i=1}^{\infty} \frac{\partial^{2} q_{i}(\tau)}{\partial \tau^{2}} \int_{0}^{1} \tilde{q}_{j}(z) \tilde{q}_{i}(z) \mathrm{d} z+\lambda\left(\frac{\omega_{f}}{\Omega}\right) \int_{0}^{1} \tilde{q}_{j}(z)\left[\left(\sum_{i=1}^{\infty} q_{i}(\tau) \tilde{q}_{i}(z)\right)^{2} \sum_{i=1}^{\infty} \frac{\partial q_{i}(\tau)}{\partial \tau} \tilde{q}_{i}(z)\right] \mathrm{d} z- \\
\lambda\left(\frac{\omega_{f}}{\Omega}\right) \sum_{i=1}^{\infty} \frac{\partial q_{i}(\tau)}{\partial \tau} \int_{0}^{1} \tilde{q}_{i}(z) \tilde{q}_{j}(z) \mathrm{d} z+\left(\frac{\omega_{f}}{\Omega}\right)^{2} \sum_{i=1}^{\infty} q_{i}(\tau) \int_{0}^{1} \tilde{q}_{j}(z) \tilde{q}_{i}(z) \mathrm{d} z=\tilde{N} \int_{0}^{1} \frac{\partial^{2} v(z, \tau)}{\partial \tau^{2}} \tilde{q}_{j}(z) \mathrm{d} z .
\end{array}
$$

Furthermore, it can be rewritten as

$$
\begin{array}{r}
\frac{\partial^{2} q_{i}(\tau)}{\partial \tau^{2}}+2 \lambda\left(\frac{\omega_{f}}{\Omega}\right) \sum_{m=1}^{\infty} \sum_{n=1}^{\infty} \sum_{p=1}^{\infty} q_{m}(\tau) q_{n}(\tau) \frac{\mathrm{d} q_{p}(\tau)}{\mathrm{d} \tau} \Phi_{m n p i}- \\
\lambda\left(\frac{\omega_{f}}{\Omega}\right) \frac{\partial q_{i}(\tau)}{\partial \tau}+\left(\frac{\omega_{f}}{\Omega}\right)^{2} q_{i}(\tau)=2 \tilde{N} \sum_{i=1}^{\infty} \frac{\partial^{2} v_{i}(\tau)}{\partial \tau^{2}} \int_{0}^{1} \tilde{v}_{i}(z) \tilde{q}_{j}(z) \mathrm{d} z
\end{array}
$$

where

$$
\Phi_{m n p i}=\int_{0}^{1} \tilde{q}_{m}(z) \tilde{q}_{n}(z) \tilde{q}_{p}(z) \tilde{q}_{i}(z) \mathrm{d} z .
$$

When $m=n=p=i=1$, Eq. (19) can be simplified as

$$
\Phi_{m n p i}=\int_{0}^{1} \tilde{q}_{1}^{4}(z) \mathrm{d} z=\int_{0}^{1} \sin ^{4}(\pi z) \mathrm{d} z=\frac{3}{8},
$$

When $i=j=1$, we introduce

$$
N=2 \tilde{N} \int_{0}^{1} \tilde{v}_{1}(z) \tilde{q}_{1}(z) \mathrm{d} z
$$

as the inertia coupling parameter, which denotes the action for the first mode motion of the structure on the first mode motion of the van der Pol oscillator. Thus the first mode motion of van der Pol oscillator can be derived as follows,

$$
\frac{\mathrm{d}^{2} q_{1}(\tau)}{\mathrm{d} \tau^{2}}+\lambda \omega_{R}\left[\frac{3}{4} q_{1}^{2}(\tau)-1\right] \frac{\mathrm{d} q_{1}(\tau)}{\mathrm{d} \tau}+\omega_{R}^{2} q_{1}(\tau)=N \frac{\mathrm{d}^{2} v_{1}(\tau)}{\mathrm{d} \tau^{2}}
$$

Eqs (16) and (21) model the interactions between the structural vibration and the van der Pol oscillation and will be investigated in the following. 


\section{Bifurcation analysis}

\subsection{The 1:1 internal resonance analysis with the multiple scale method}

The nonlinear systems display rich dynamic characteristics when the resonance occurs ${ }^{[44,45]}$, such as the primary resonance, superharmonic/subharmnic resonance and the internal resonance. The multiple scale method is a good way to understand the qualitative characteristics of the system which presents the internal resonance ${ }^{[46-48]}$. The phenomenon of lock-in or synchronization is often analyzed in the vortex-induced vibration for the long cylinders (e.g. the offshore risers), which means the vortex shedding frequency $f_{V}$ tends to the natural frequency of the structure $f_{S}{ }^{[29]}$. Similarly, the phenomenon for frequencies approximation could also be encountered in the coupled system proposed in this paper. For Eqs (16) and (21), the nondimensional frequencies $\omega_{s}$ and $\omega_{R}$ of the first-order coupled system, as shown in Fig.3, are approximate to each other around the rotating speed $\Omega \approx 450 \mathrm{rad} / \mathrm{s}$.

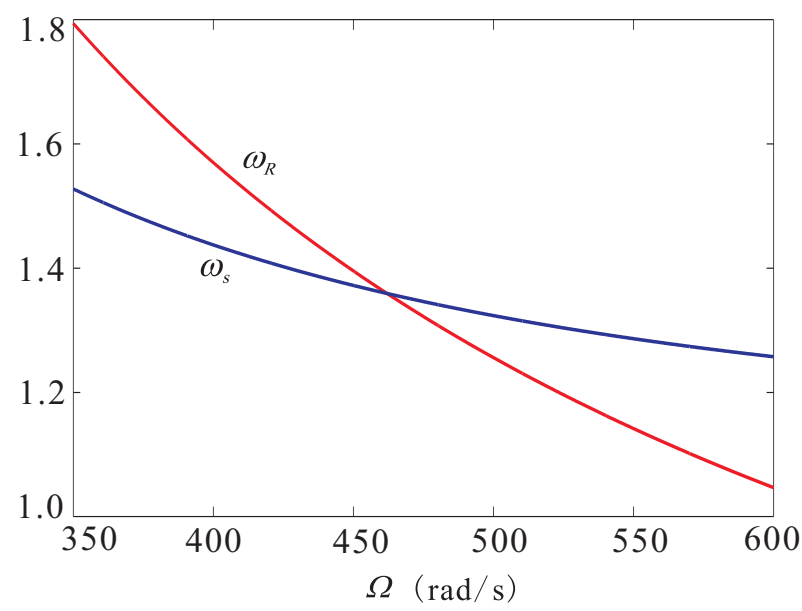

Fig. 3 The non-dimensional frequencies $\omega_{s}$ and $\omega_{R}$ varying with the rotating speed $\Omega$.

Hence the 1:1 internal resonance of the coupled system is interesting, which is investigated by using the multiple scale method in the following. The relation between the non-dimensional frequencies $\omega_{s}$ and $\omega_{R}$ can be written as $\omega_{s}=\omega_{R}+\varepsilon \sigma$, where $\sigma$ is the detuning parameter. Introducing the scaling parameters $\zeta \rightarrow \varepsilon \zeta, C_{L 0} \rightarrow \varepsilon C_{L 0}, N \rightarrow \varepsilon N, \lambda \rightarrow \varepsilon \lambda$ into Eqs (16) and (21), one can be obtain that

$$
\begin{array}{r}
\ddot{v}_{1}+\omega_{s}^{2} v_{1}=\varepsilon\left(b \omega_{R}^{2}+d\right) q_{1}-\varepsilon \zeta \dot{v}_{1}, \\
\ddot{q}_{1}+\omega_{R}^{2} q_{1}=\varepsilon N \ddot{v}_{1}-\varepsilon \lambda \omega_{R}\left(\frac{3}{4} q_{1}^{2}-1\right) \dot{q}_{1} .
\end{array}
$$

Assume the approximate form of the solutions as follows,

$$
\begin{gathered}
v_{1}(\tau)=v_{10}\left(T_{0}, T_{1}\right)+\varepsilon v_{11}\left(T_{0}, T_{1}\right)+\cdots, \\
q_{1}(\tau)=q_{10}\left(T_{0}, T_{1}\right)+\varepsilon q_{11}\left(T_{0}, T_{1}\right)+\cdots .
\end{gathered}
$$

Substituting (23) into Eq. (22) and equating coefficient of like powers of $\varepsilon$, one can obtain that:

Order $\varepsilon^{0}$,

$$
\begin{aligned}
& D_{0}^{2} v_{10}+\omega_{s}^{2} v_{10}=0, \\
& D_{0}^{2} q_{10}+\omega_{R}^{2} q_{10}=0 .
\end{aligned}
$$


Order $\varepsilon^{1}$

$$
\begin{array}{r}
D_{0}^{2} v_{11}+\omega_{s}^{2} v_{11}=\left(b \omega_{R}^{2}+d\right) q_{10}-\zeta D_{0} v_{10}-2 D_{0} D_{1} v_{10} \\
D_{0}^{2} q_{11}+\omega_{R}^{2} q_{11}=N D_{0}^{2} v_{10}-\lambda \omega_{R}\left(\frac{3}{4} q_{10}^{2}-1\right) D_{0} q_{10}-2 D_{0} D_{1} q_{10}
\end{array}
$$

where $\frac{\mathrm{d}}{\mathrm{d} \tau}=D_{0}+\varepsilon D_{1}+\varepsilon^{2} D_{2}+\cdots, \frac{\mathrm{d}^{2}}{\mathrm{~d} \tau^{2}}=D_{0}^{2}+2 \varepsilon D_{0} D_{1}+\cdots$, and $D_{n}=\frac{\partial}{\partial T_{n}}$ is the partial differential operator. The solutions of Eqs (24) and (25) can be obtained as

$$
\begin{gathered}
v_{10}=A\left(T_{1}\right) \mathrm{e}^{\mathrm{i} \omega_{s} \tau}+\bar{A}\left(T_{1}\right) \mathrm{e}^{-\mathrm{i} \omega_{s} \tau}, \\
q_{10}=B\left(T_{1}\right) \mathrm{e}^{\mathrm{i} \omega_{R} \tau}+\bar{B}\left(T_{1}\right) \mathrm{e}^{-\mathrm{i} \omega_{R} \tau} .
\end{gathered}
$$

Substitute (28) and (29) into Eqs (26) and (27) and consider the internal resonance condition, yielding

$$
\begin{array}{r}
D_{0}^{2} v_{11}+\omega_{s}^{2} v_{11}=\left(b \omega_{R}^{2}+d\right) B \mathrm{e}^{\mathrm{i}\left(\omega_{s}-\varepsilon \sigma\right) \tau}-\mathrm{i} \omega_{s} \zeta A \mathrm{e}^{\mathrm{i} \omega_{s} \tau}-2 \mathrm{i} \omega_{s} \mathrm{e}^{\mathrm{i} \omega_{s} \tau} D_{1} A+c . c ., \\
D_{0}^{2} q_{11}+\omega_{R}^{2} q_{11}=-\omega_{s}^{2} N A \mathrm{e}^{\mathrm{i}\left(\omega_{R}+\varepsilon \sigma\right) \tau}-\mathrm{i} \lambda \omega_{R}^{2}\left[\frac{3}{4} B^{3} \mathrm{e}^{3 \mathrm{i} \omega_{R} \tau}+\left(\frac{3}{4} B \bar{B}-1\right) B \mathrm{e}^{\mathrm{i} \omega_{R} \tau}\right] \\
-2 \mathrm{i} \omega_{R} D_{1} B \mathrm{e}^{\mathrm{i} \omega_{R} \tau}+c . c
\end{array}
$$

where c.c. stands for the complex conjugate of the proceeding terms.

The solvable conditions of Eqs (30) and (31) are derived by equating the coefficients of secular terms to be zero, that is

$$
\begin{array}{r}
\left(b \omega_{R}^{2}+d\right) B \mathrm{e}^{-\mathrm{i} \sigma T_{1}}-\mathrm{i} \omega_{s} \zeta A-2 \mathrm{i} \omega_{s} D_{1} A=0, \\
-\omega_{s}^{2} N A \mathrm{e}^{\mathrm{i} \sigma T_{1}}-\mathrm{i} \lambda \omega_{R}^{2}\left(\frac{3}{4} B \bar{B}-1\right) B-2 \mathrm{i} \omega_{R} D_{1} B=0 .
\end{array}
$$

The derivatives of amplitudes $A$ and $B$ with respect to $T_{1}$ can be obtained by Eqs (32) and (33), which reads

$$
\begin{aligned}
D_{1} A & =\frac{-1}{2 \omega_{s}}\left[\mathrm{i}\left(b \omega_{R}^{2}+d\right) B \mathrm{e}^{-\mathrm{i} \sigma T_{1}}+\omega_{s} \zeta A\right], \\
D_{1} B & =\frac{1}{2 \omega_{R}}\left[\mathrm{i} \omega_{s}^{2} N A \mathrm{e}^{\mathrm{i} \sigma T_{1}}-\lambda \omega_{R}^{2}\left(\frac{3}{4} B \bar{B}-1\right) B\right] .
\end{aligned}
$$

The functions $A$ and $B$ can be expressed in the polar co-ordinates as

$$
A\left(T_{1}\right)=\frac{a_{1}\left(T_{1}\right)}{2} \mathrm{e}^{\mathrm{i} \theta_{1}\left(T_{1}\right)}, B\left(T_{1}\right)=\frac{a_{2}\left(T_{1}\right)}{2} \mathrm{e}^{\mathrm{i} \theta_{2}\left(T_{1}\right)},
$$

where $a_{j}, \theta_{j}(j=1,2)$ are the amplitudes and phase angles, respectively. By substituting (36) into Eqs (34) and (35), the first-order differential equations can be obtained after separating the real and imaginary parts:

$$
\begin{aligned}
a_{1}^{\prime} & =\frac{1}{2 \omega_{s}}\left[\left(b \omega_{R}^{2}+d\right) a_{2} \sin (\varphi)-\omega_{s} \zeta a_{1}\right] \\
\theta_{1}^{\prime} & =\frac{-\left(b \omega_{R}^{2}+d\right) a_{2} \cos (\varphi)}{2 a_{1} \omega_{s}}, \\
a_{2}^{\prime} & =\frac{\omega_{s}^{2} N a_{1} \sin (\varphi)}{2 \omega_{R}}-\frac{\lambda \omega_{R}\left(3 a_{2}^{2}-16\right) a_{2}}{32} \\
\theta_{2}^{\prime} & =\frac{N \omega_{s}^{2} a_{1}}{2 a_{2} \omega_{R}} \cos (\varphi),
\end{aligned}
$$


where $\left({ }^{\prime}\right)$ denotes the derivatives with respect to $T_{1}$, and $\varphi=\theta_{2}-\sigma T_{1}-\theta_{1}$. The derivative of $\varphi$ with respect to $T_{1}$ can be obtained by eliminating $\theta_{1}$ and $\theta_{2}$ from Eqs (38) and (40), which reads

$$
\varphi^{\prime}=\frac{N \omega_{s}^{2} a_{1}}{2 a_{2} \omega_{R}} \cos \varphi-\sigma+\frac{\left(b \omega_{R}^{2}+d\right)}{2 a_{1} \omega_{s}} a_{2} \cos \varphi .
$$

The equilibrium solutions of Eqs (37), (39) and (41) correspond to periodic motions of the coupled system. To obtain the equilibrium solutions, $a_{j}^{\prime}(j=1,2)$ and $\varphi^{\prime}$ in Eqs (37), (39) and (41) are assumed to equal to zero. One can obtain the frequency-response equations as follows

$$
\begin{gathered}
\left(b \omega_{R}^{2}+d\right) \lambda \omega_{R}^{2}\left(\frac{3 a_{2}^{2}}{16}-1\right) a_{2}^{2}-\omega_{s} \zeta \omega_{s}^{2} N a_{1}^{2}=0 \\
{\left[\frac{N \omega_{s}^{2} a_{1}}{2 a_{2} \omega_{R}}+\frac{\left(b \omega_{R}^{2}+d\right) a_{2}}{2 a_{1} \omega_{s}}\right]^{2}=\sigma^{2}+\left[\frac{\zeta}{2}+\frac{\lambda \omega_{R}}{2}\left(\frac{3 a_{2}^{2}}{16}-1\right)\right]^{2}}
\end{gathered}
$$

Eqs (42) and (43) can reveal the effects of system parameters on the responses.

\subsection{Singularity analysis for the steady-state responses}

The structural motion and the van der Pol damping have important influence on the vibration of the fluid. Then the fluid motion in turn affects the structural vibration. In order to investigate the bifurcation characteristics of the coupled system in a wider parameter space, an engineering unfolding analysis is carried out, see details shown in [49-51]. Eqs (42) and (43) can be rewritten as

$$
\begin{gathered}
G_{1}\left(a_{1}, a_{2}\right)=\left(b \omega_{R}^{2}+d\right) \lambda \omega_{R}^{2}\left(\frac{3 a_{2}^{2}}{16}-1\right) a_{2}^{2}-\zeta \omega_{s}^{3} N a_{1}^{2}=0, \\
G_{2}\left(a_{1}, a_{2}, \sigma\right)=4 \sigma^{2} \omega_{R}^{3} \omega_{s}^{4} a_{1}^{2} a_{2}^{2}+\omega_{R}^{3} \omega_{s}^{4}\left(\zeta-\lambda \omega_{R}\right)^{2} a_{1}^{2} a_{2}^{2}+\frac{9 \lambda^{2}}{256} \omega_{R}^{4} \omega_{s}^{2} a_{1}^{2} a_{2}^{6}-N^{2} \omega_{s}^{2} \omega_{s}^{4} a_{1}^{4} \\
+\frac{3 \lambda}{8}\left(\zeta-\lambda \omega_{R}\right) \omega_{s}^{2} \omega_{R}^{3} a_{1}^{2} a_{2}^{4}-2 \omega_{R} \omega_{s}^{3}\left(b \omega_{R}^{2}+d\right) N a_{1}^{2} a_{2}^{2}-\omega_{R}^{2}\left(b \omega_{R}^{2}+d\right)^{2} a_{2}^{4}=0,
\end{gathered}
$$

of which $\sigma$ is chosen as the bifurcation parameter, $\lambda$ and $N$ as the engineering unfolding parameters, $a_{1}$ and $a_{2}$ as the state variables. The transition set is derived in the following form by using the singularity method, for details referring to [52].

$$
\begin{gathered}
\qquad=B \cup H \cup D \\
B=\left\{\begin{array}{l}
(\lambda, N) \in R^{2}: \exists(z, \sigma), \text { s.t. } \\
G_{1}(z, \lambda, N)=0, G_{2}(z, \sigma, \lambda, N)=0 \\
G_{1 a_{1}} G_{2 a_{2}}-G_{1 a_{2}} G_{2 a_{1}}=0, G_{1 a_{2}} G_{2 \sigma}-G_{2 a_{2}} G_{1 \sigma}=0, z=\left(a_{1}, a_{2}\right),
\end{array}\right. \\
H=\left\{\begin{array}{l}
(\lambda, N) \in R^{2}: \exists(z, \sigma), \text { s.t. } \\
G_{1}=0, G_{2}=0, G_{1 a_{1}} G_{2 a_{2}}-G_{1 a_{2}} G_{2 a_{1}}=0, \\
\left(G_{1 a_{1}, a_{1}} G_{1 a_{2}}^{2}+G_{1 a_{1}}^{2} G_{1 a_{2}, a_{2}}-2 G_{1 a_{1}, a_{2}} G_{1 a_{1}} G_{1 a_{2}}\right) G_{2 a_{1}}- \\
\left(G_{2 a_{1}, a_{1}} G_{1 a_{2}}^{2}+G_{1 a_{1}}^{2} G_{2 a_{2}, a_{2}}-2 G_{2 a_{1}, a_{2}} G_{1 a_{1}} G_{1 a_{2}}\right) G_{1 a_{1}}=0, z=\left(a_{1}, a_{2}\right)
\end{array}\right. \\
D=\left\{\begin{array}{l}
(\lambda, N) \in R^{2}: \exists\left(z_{1}, z_{2}, \sigma\right), \text { s.t. } z_{1} \neq z_{2}, \\
G_{1}=0, G_{2}=0, \operatorname{det}(\mathrm{d} G)_{z_{i}, \sigma, \lambda, N 1}=0, \\
z=\left(a_{1}, a_{2}\right), \quad i=1,2,
\end{array}\right.
\end{gathered}
$$

where $B, H$ and $D$ represent the bifurcation set, hysteresis set and double limit set, respectively. The partial derivatives of the bifurcation functions $G_{1}$ and $G_{2}$ with respect to $\sigma, a_{1}$ and $a_{2}$ are calculated to obtain the bifurcation sets, see Appendix for details. Thus the transition set of the coupled system is derived by solving the algebraic equations shown in (47)-(49). According 
to the analysis in the literature ${ }^{[17,25,43]}$, the other parameters are fixed at $A=4.2 \times 10^{-4} \mathrm{~m}^{2}$, $\rho=7800 \mathrm{~kg} / \mathrm{m}^{3}, \rho_{f}=1.225 \mathrm{~kg} / \mathrm{m}^{3}, E I=300 \mathrm{~N} \cdot \mathrm{m}, V=100 \mathrm{~m} / \mathrm{s}, \Omega=450 \mathrm{rad} / \mathrm{s}, D_{0}=0.1 \mathrm{~m}$, $L=0.3 \mathrm{~m}, c=6 \mathrm{~N} \cdot \mathrm{s} / m, \mathrm{St}=0.1, C_{L 0}=0.3$.

It can be seen from Fig. 4 that the two-parameter space is divided into twelve parts by the transition set, where the red lines denote the bifurcation set and the blue lines present the hysteresis set. Herein the double limit set is $D=\emptyset$ after calculation.

The transition set can be used to classify different kinds of responses of the system. The responses of the coupled system can display different dynamic characteristics when the van der Pol damping $\lambda$ and the coupling parameter $N$ are chosen in different parameter subspaces.

The representative frequency-response curves of the system in each region of the twoparameter space as shown in Fig.4 are computed, and the stability is determined by examining the eigenvalues of the corresponding characteristic equation for Eqs (37), (39) and (41). The characteristic equation is a triple polynomial, which reads

$$
\lambda^{3}+p_{1} \lambda^{2}+p_{2} \lambda+p_{3}=0 .
$$

A solution is stable if all of eigenvalues have negative real part, which is decided by the RouthHurwitz criterion ${ }^{[53]}$ that: $p_{1}>0, p_{3}>0$ as well as $p_{1} p_{2}-p_{3}>0$.

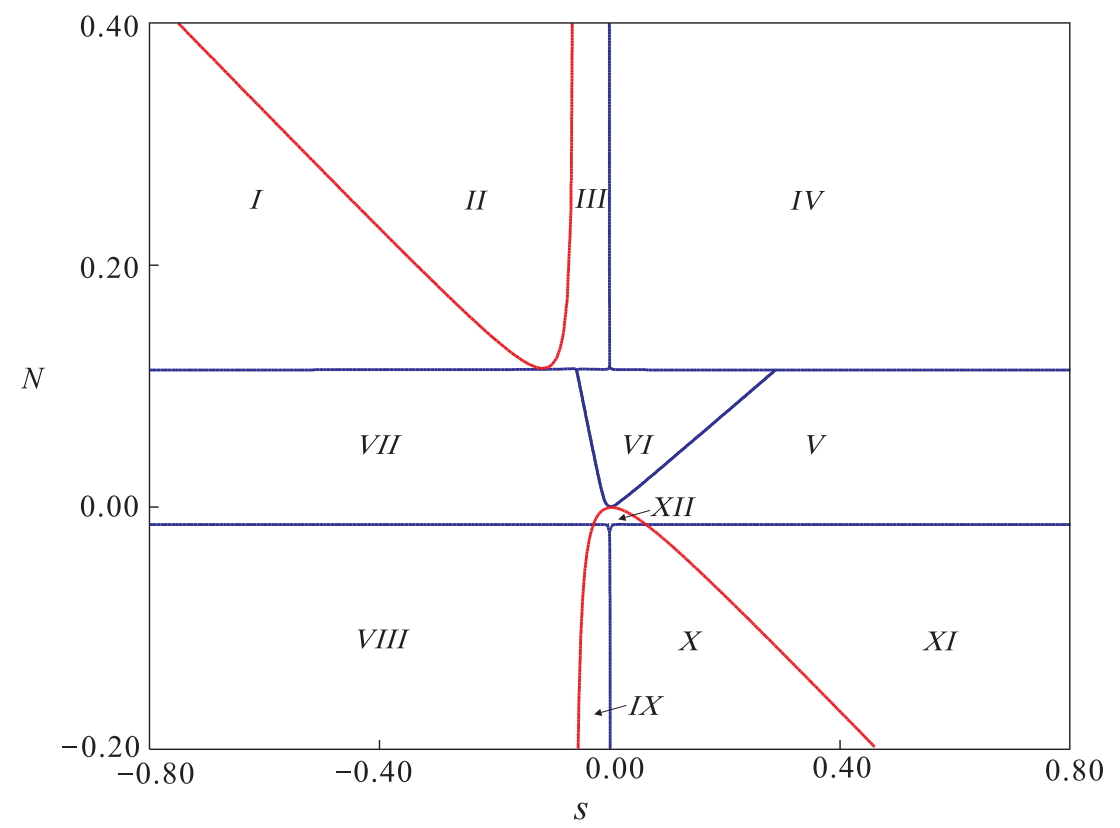

Fig. 4 The transition set: the red and blue lines denote the bifurcation and hysteresis sets, respectively.

Fig. 5 shows that the amplitudes $a_{1}$ and $a_{2}$ of the steady-state solutions are unstable for $\lambda=-0.0639$ and $N=0.1836$ in Region $I$, and the varying trends of the amplitudes $a_{1}$ and $a_{2}$ with respect to the detuning parameter $\sigma$ are opposite to each other.

Fig.6 depicts that the trivial solutions of the amplitudes $a_{1}$ and $a_{2}$ lose stabilities via a saddle-node bifurcation at $S N_{1}$, resulting in the occurrence of a two-mode solution. One of the two-mode solution is unstable for both the amplitudes $a_{1}$ and $a_{2}$ and it has the opposite varying trend for amplitudes $a_{1}$ and $a_{2}$ with respect to the detuning parameter $\sigma$. The other one of the two-mode solution is unstable until a Hopf bifurcation occurs at $H_{1}$, resulting in the change of the unstable solutions into stable ones. The stable solutions decrease until encounter 

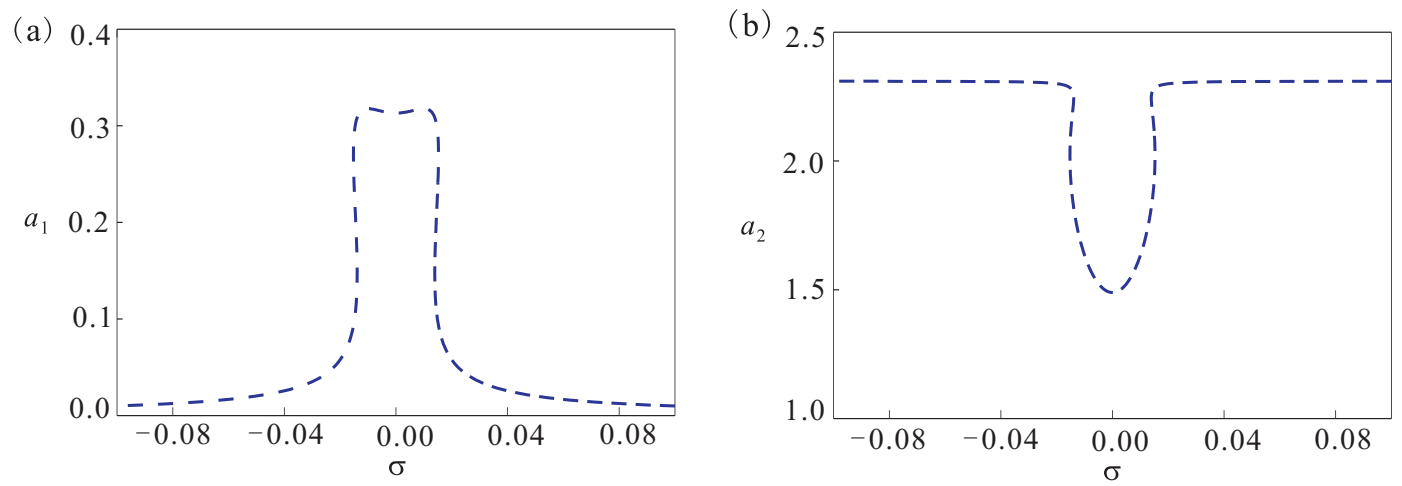

Fig. 5 Frequency-response curves in Region $I$ when $\lambda=-0.0639, N=0.1836$ : (a) the amplitude $a_{1}$ with respect to the detuning parameter $\sigma$; (b) the amplitude $a_{2}$ with respect to the detuning parameter $\sigma$.

another saddle-node bifurcation at $S N_{2}$, leading to a change for the solutions from stable to unstable. Then the unstable solutions decrease until another Hopf bifurcation occurs at $H_{2}$, resulting in a change of the unstable solutions to the stable trivial ones.
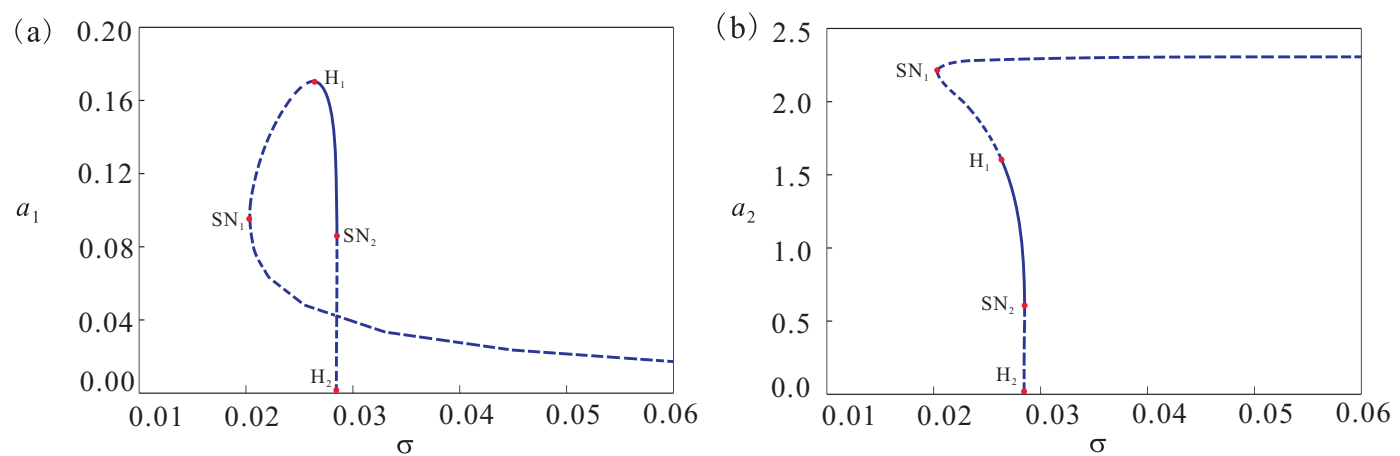

Fig. 6 Frequency-response curves in Region $I I$ when $\lambda=-0.0372, N=0.3720$ : (a) the amplitude $a_{1}$ with respect to the detuning parameter $\sigma$; (b) the amplitude $a_{2}$ with respect to the detuning parameter $\sigma$.

Fig. 7 shows that as the detuning parameter $\sigma$ increases, the trivial solutions jump to the large unstable solutions via a Hopf bifurcation $(\mathrm{H})$ at $\sigma=0.0183$. Then the unstable amplitude $a_{1}$ decreases while $a_{2}$ increases as $\sigma$ increases. As $\sigma$ decreases, the unstable solutions jump to the trivial ones via a saddle-node bifurcation $(\mathrm{SN})$ at $\sigma=0.0181$, leading to the change of the solutions from unstable to stable.

It can be seen from Fig.8 that as $\sigma$ increases, the responses increase until encounter a saddle-node bifurcation at $S N_{1}$, resulting in the responses jump to the large solutions. The large responses increase until reach the maximum values, then decrease until a saddle-node bifurcation occurs at $S N_{2}$, leading to the solutions jump to the small solutions, then the responses decrease all the way as $\sigma$ increases. Similarly, as $\sigma$ decreases, the amplitudes $a_{1}$ and $a_{2}$ have the same varying trends and encounter two saddle-node bifurcations at $S N_{3}$ and $S N_{4}$, respectively.

Fig.9 shows that the amplitudes of the steady-state solutions of the beam and van der Pol oscillator are stable for $\lambda=0.0400$ and $N=0.0519$. Both Fig.8 and Fig.9 show that the varying trend of the amplitude $a_{1}$ with respect to the detuning parameter is as same as that of the amplitude $a_{2}$. 

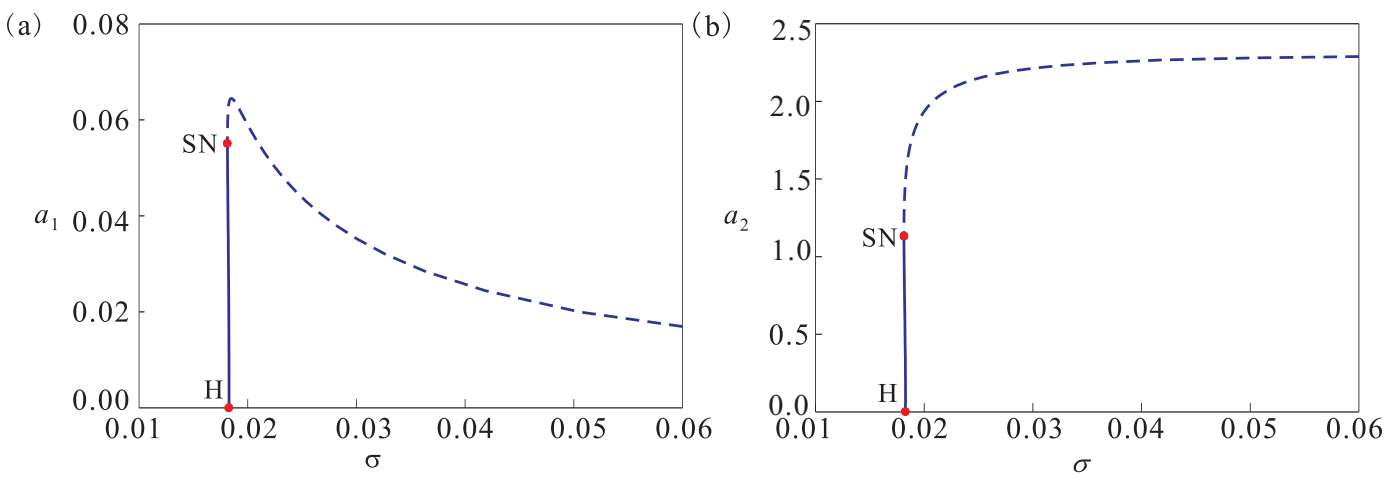

Fig. 7 Frequency-response curves in Region $I I I$ when $\lambda-0.0043, N=0.3000$ : (a) the amplitude $a_{1}$ with respect to the detuning parameter $\sigma$; (b) the amplitude $a_{2}$ with respect to the detuning parameter $\sigma$.
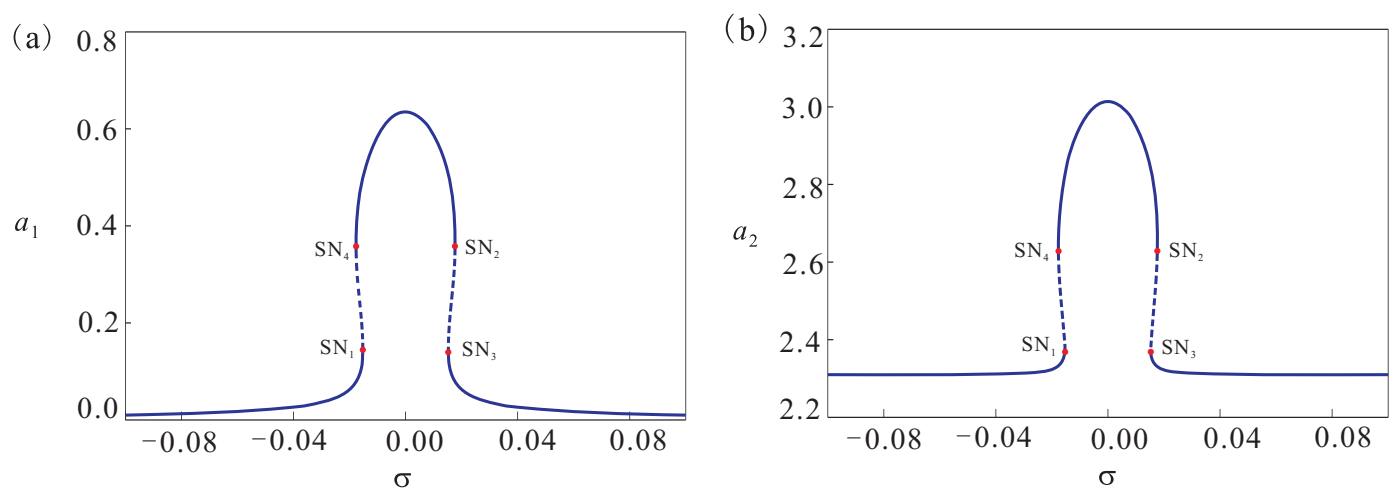

Fig. 8 Frequency-response curves in Region $I V$ when $\lambda=0.0629, N=0.2178$ : (a) the amplitude $a_{1}$ with respect to the detuning parameter $\sigma$; (b) the amplitude $a_{2}$ with respect to the detuning parameter $\sigma$.
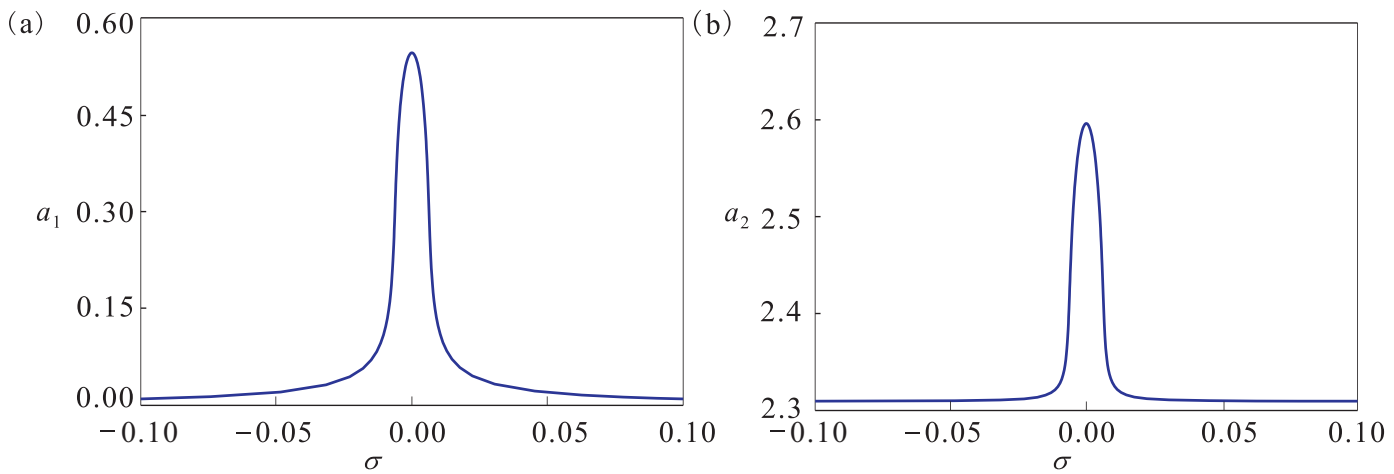

Fig. 9 Frequency-response curves in Region $V$ when $\lambda=0.0400, N=0.0519$ : (a) the amplitude $a_{1}$ with respect to the detuning parameter $\sigma$; (b) the amplitude $a_{2}$ with respect to the detuning parameter $\sigma$.

It can be seen from Fig.10 that the stable trivial solutions become unstable ones via a Hopf bifurcation for $\sigma=0.0090$. 

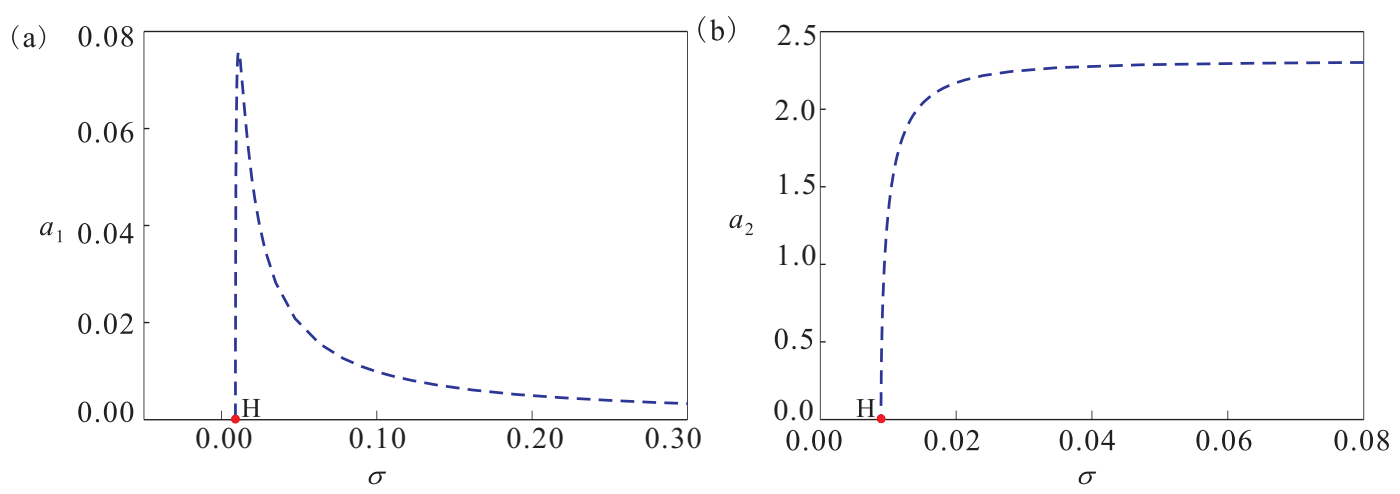

Fig. 10 Frequency-response curves in Region $V I$ when $\lambda=-0.0013, N=0.0655$ : (a) the amplitude $a_{1}$ with respect to the detuning parameter $\sigma$; (b) the amplitude $a_{2}$ with respect to the detuning parameter $\sigma$.

Figs11-13 show that the solutions in Regions VII-IX are unstable solutions expect the trivial solutions. While the varying trends of the amplitudes $a_{1}$ and $a_{2}$ with respect to the detuning parameter $\sigma$ are opposite in Fig.11 and same in Figs12 and 13.
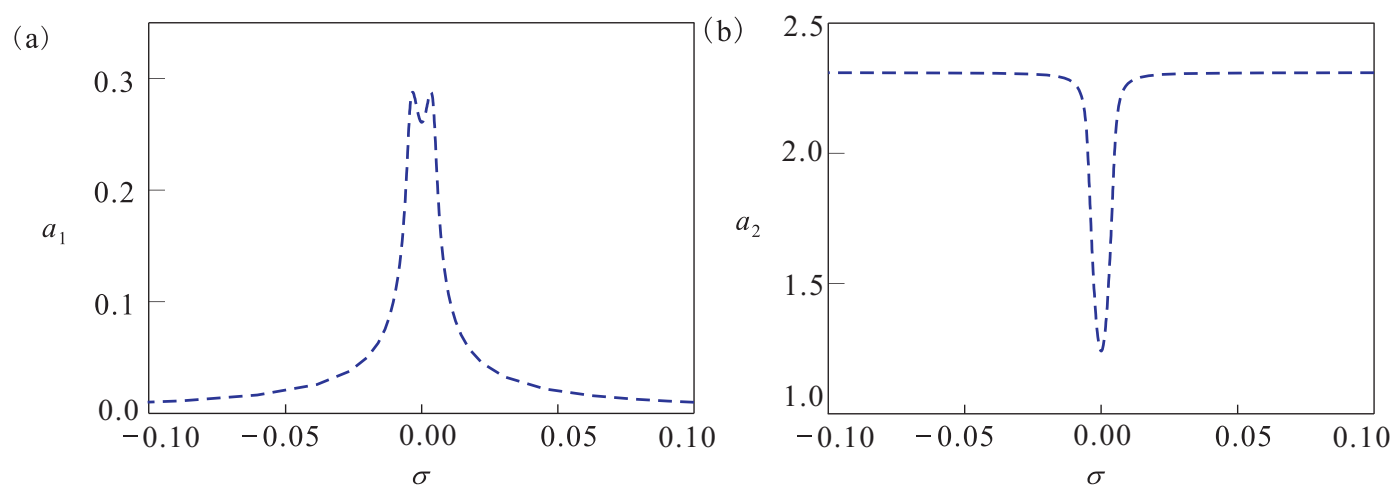

Fig. 11 Frequency-response curves in Region VII when $\lambda=-0.0090, N=0.0315$ : (a) the amplitude $a_{1}$ with respect to the detuning parameter $\sigma$; (b) the amplitude $a_{2}$ with respect to the detuning parameter $\sigma$.

It can be seen from Fig.14 that the trivial solutions jump to the large stable solutions via a Hopf bifurcation $\left(H_{1}\right)$ at $\sigma=-0.0015$ as $\sigma$ increases. Then the amplitude $a_{1}$ decreases and the amplitude $a_{2}$ increases to the trivial solutions as $\sigma$ increases. While as $\sigma$ decreases, the amplitude $a_{1}$ increases and the amplitude $a_{2}$ decreases until a Hopf bifurcation $\left(H_{2}\right)$ occurs at $\sigma=-0.0052$, leading to the change of the stable solutions to unstable ones. Decreasing $\sigma$ beyond $H_{2}$, the amplitudes $a_{1}$ and $a_{2}$ jump to the trivial solutions as $\sigma$ decreases.

It can be seen from Fig. 15 that as $\sigma$ increases, the amplitude $a_{1}$ grows while the amplitude $a_{2}$ decreases all the way until a Hopf bifurcation $\left(H_{1}\right)$ occurs at $\sigma=0.0045$, after that, the responses encounter a saddle-node bifurcation $\left(S N_{1}\right)$ at $\sigma=0.0064$, resulting in a change of the stable solutions to unstable ones. Beyond $S N_{1}$, the amplitude $a_{1}$ jumps to the small solution while $a_{2}$ jumps to the large solution. Then the amplitude $a_{1}$ decreases while $a_{2}$ increases all the way as $\sigma$ increases. The amplitudes $a_{1}$ and $a_{2}$ have the symmetry varying trends as $\sigma$ decreases.

Fig.16 shows that the trivial solutions jump to the stable solutions via a Hopf bifurcation for 

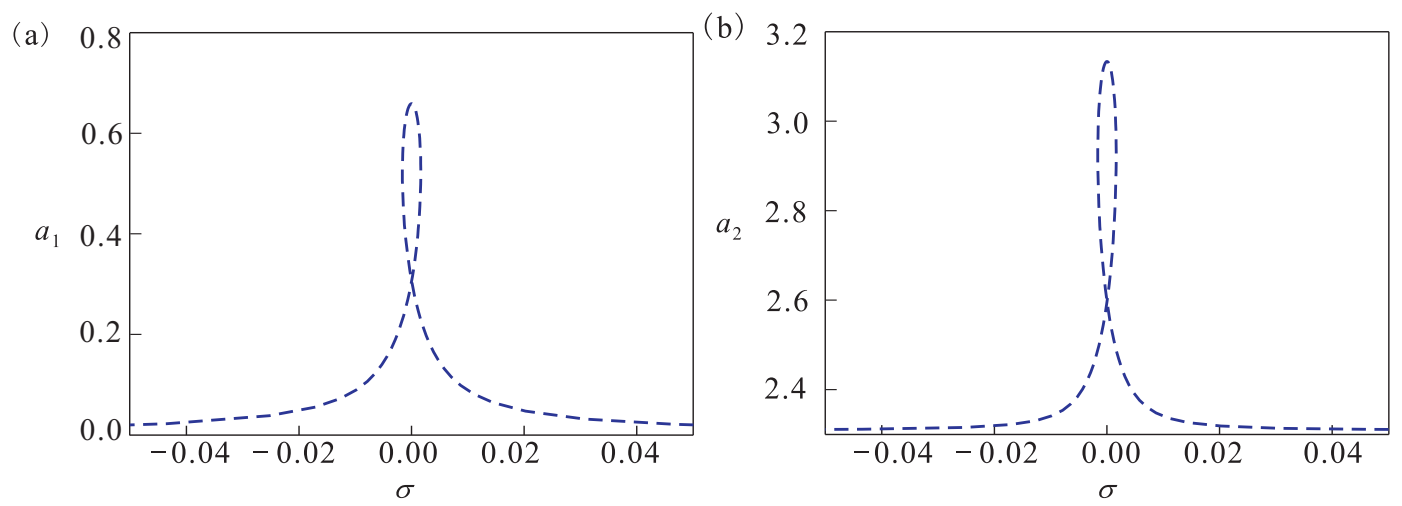

Fig. 12 Frequency-response curves in Region VIII when $\lambda=-0.0110, N=-0.0455$ : (a) the amplitude $a_{1}$ with respect to the detuning parameter $\sigma$; (b) the amplitude $a_{2}$ with respect to the detuning parameter $\sigma$.
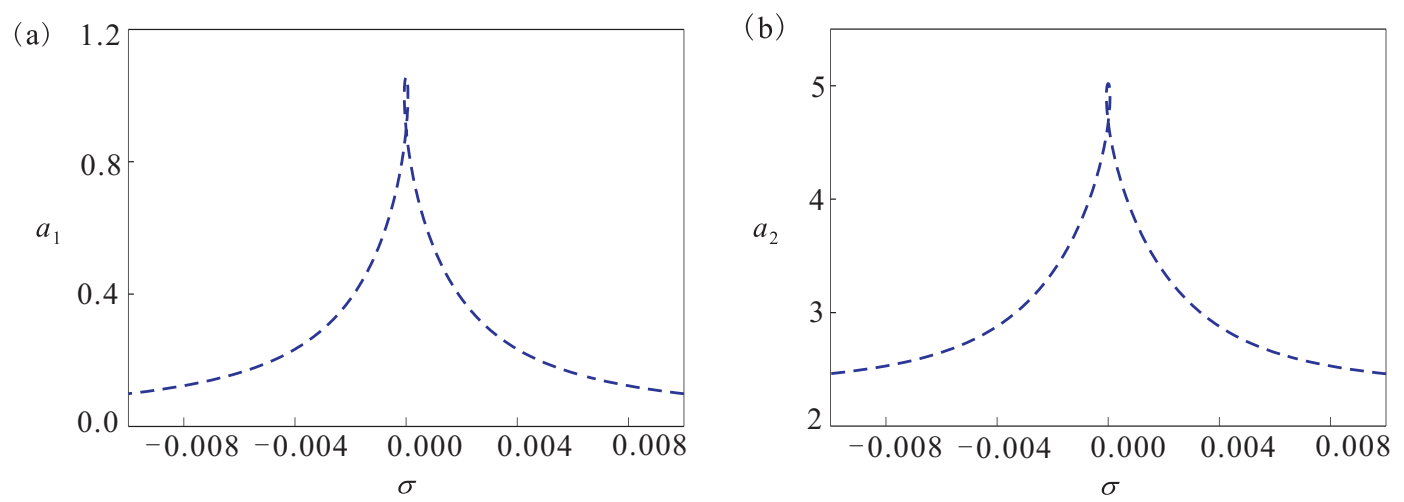

Fig. 13 Frequency-response curves in Region $I X$ when $\lambda=-0.0009, N=-0.0172$ : (a) the amplitude $a_{1}$ with respect to the detuning parameter $\sigma$; (b) the amplitude $a_{2}$ with respect to the detuning parameter $\sigma$.
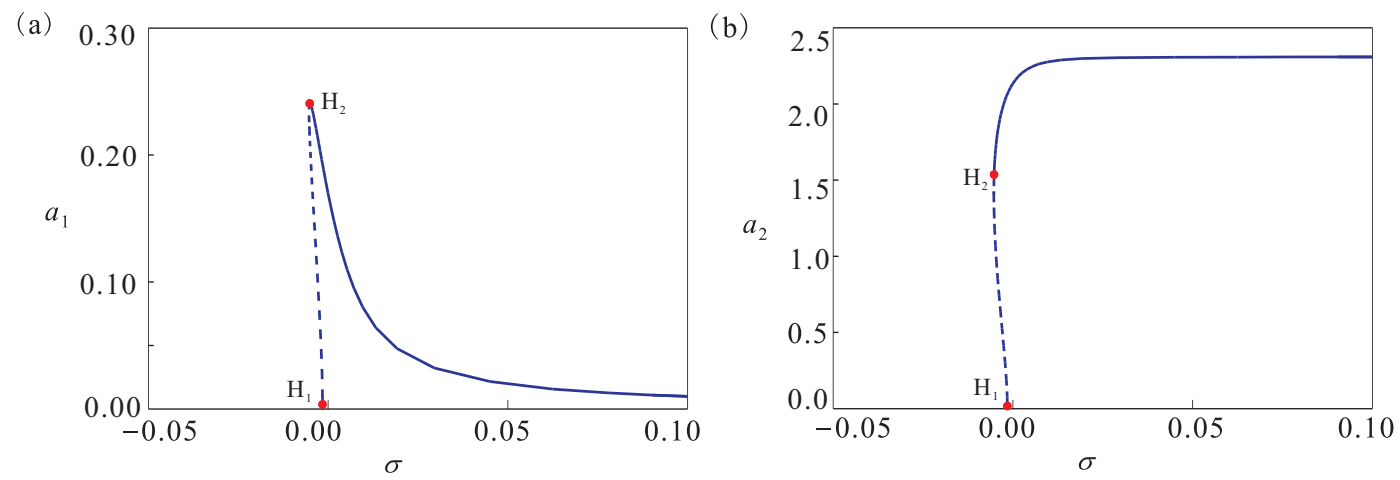

Fig. 14 Frequency-response curves in Region $X$ when $\lambda=0.0200, N=-0.1000$ : (a) the amplitude $a_{1}$ with respect to the detuning parameter $\sigma$; (b) the amplitude $a_{2}$ with respect to the detuning parameter $\sigma$.

$\sigma=0.0003$. The amplitude $a_{1}$ jump to the maximum values rapidly. Then as $\sigma$ increases, the 

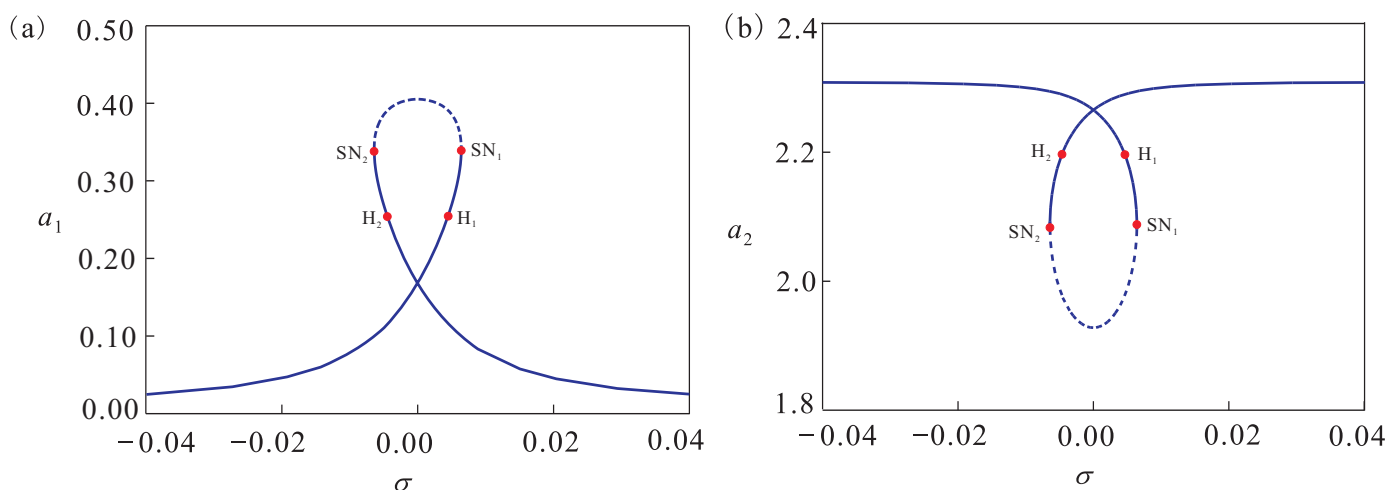

Fig. 15 Frequency-response curves in Region $X I$ when $\lambda=0.0780, N=-0.1163$ : (a) the amplitude $a_{1}$ with respect to the detuning parameter $\sigma$; (b) the amplitude $a_{2}$ with respect to the detuning parameter $\sigma$.

amplitude $a_{1}$ decreases while the amplitude $a_{2}$ increases gradually until to the trivial solutions.
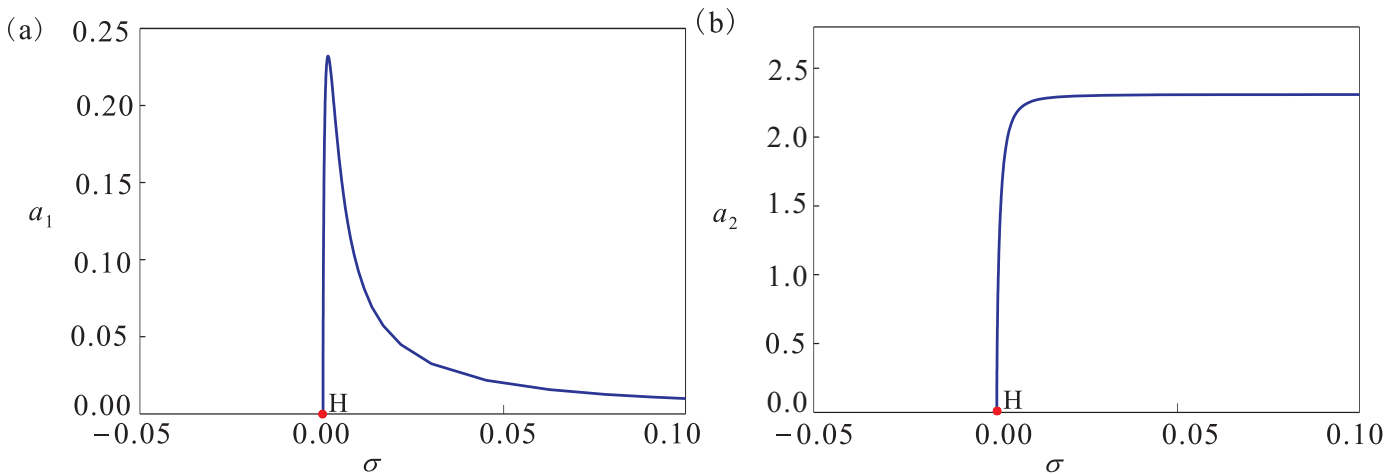

Fig. 16 Frequency-response curves in Region $X I I$ when $\lambda=0.0013, N=-0.0070$ : (a) the amplitude $a_{1}$ with respect to the detuning parameter $\sigma$; (b) the amplitude $a_{2}$ with respect to the detuning parameter $\sigma$.

The results show that when parameters $\lambda$ and $N$ have the same signs, that is $\lambda N>0$, then the varying trends for the structural and van der Pol vibrations are same to each other with respect to the detuning parameter $\sigma$. Specifically, when $\lambda>0$ and $N>0$, the solutions are stable or can encounter the saddle-node bifurcation as $\sigma$ varying; when $\lambda<0$ and $N<0$, the solutions are unstable as $\sigma$ varying. While for parameters $\lambda$ and $N$ have the opposite signs, that is $\lambda N<0$, the varying trends of the two coupled motions are reverse to each other, which means the energy transfer between the two modes. The Hopf bifurcation can occur for certain parameter values when $\lambda N<0$.

\section{Numerical results}

The response bifurcations of the coupled system including the Hopf and saddle-node bifurcations can occur under certain parameter values as analyzed in the previous section. In order to verify the validity of the multiple scale method, characteristics of dynamic responses for the original systems $(16)$ and $(21)$ are investigated. A representative point $(\sigma=0.0285)$ after 
the Hopf bifurcation as shown in Fig.6 is chosen with the unfolding parameters $\lambda=-0.0372$ and $N=0.3720$. The other parameters are fixed at $A=4.2 \times 10^{-4} \mathrm{~m}^{2}, \rho=7800 \mathrm{~kg} / \mathrm{m}^{3}$, $\rho_{f}=1.225 \mathrm{~kg} / \mathrm{m}^{3}, E I=300 \mathrm{~N} \cdot \mathrm{m}, V=100 \mathrm{~m} / \mathrm{s}, \Omega=450 \mathrm{rad} / \mathrm{s}, D_{0}=0.1 \mathrm{~m}, L=0.3 \mathrm{~m}$, $c=6 \mathrm{~N} \cdot \mathrm{s} / m, \mathrm{St}=0.1, C_{L 0}=0.3$. The time histories, phase portraits and the Lyapunov exponents for the original structural and van der Pol motions with the above parameter values are computed by using the Runge-Kutta method as shown in Figs 17 and 18. It shows that the largest Lyapunov exponent of the original system with the initial condition values: $\left[v_{1}(0)=0.05, y(0)=0.00, q_{1}(0)=0.50, p(0)=0.00\right]$ (assuming $\dot{v}_{1}(\tau)=y(\tau)$ and $\dot{q}_{1}(\tau)=p(\tau)$ ) tends to zero as time $\tau$ increases, which means the quasi-periodic solutions occurring under this parameter conditions. Thus the Hopf bifurcation for the modulated solutions indicates the occurrence of quasi-periodic solutions for the original system, which can result in important physical consequences ${ }^{[54]}$, such as a behaviour from transition to chaos. The saddle-node

(a)

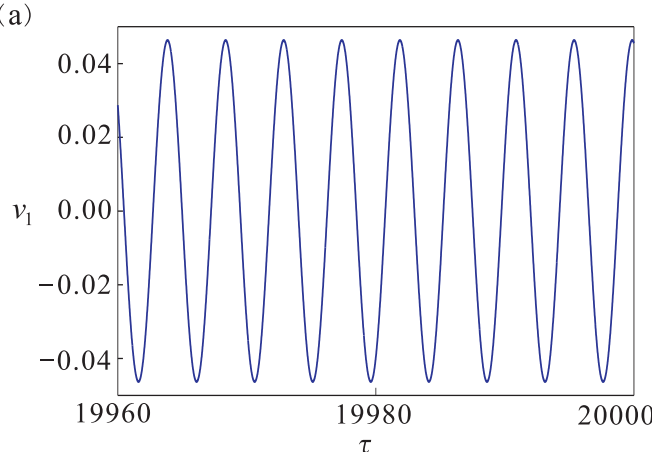

(c)

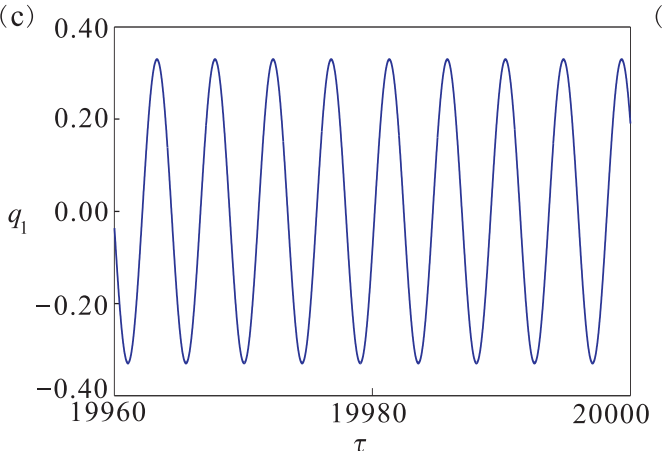

(b)

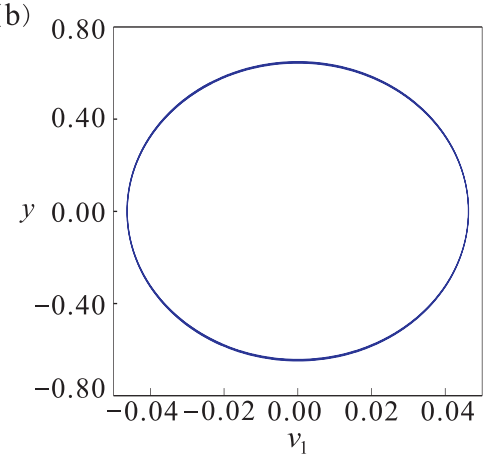

(d)

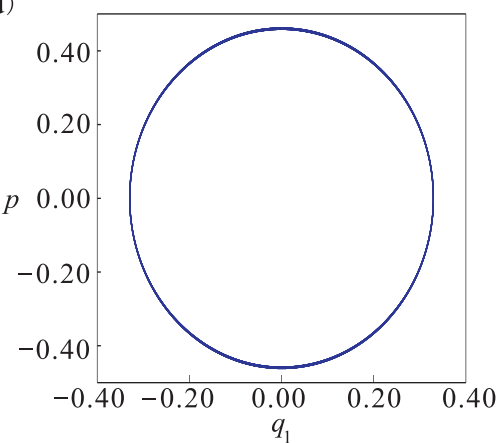

Fig. 17 Time histories and phase portraits of vibrations for the original system at a typical point $(\sigma=0.0285)$ after the Hopf bifurcation as shown in Figure 6 with the initial conditions $\left[v_{1}(0)=0.05, y(0)=0.00, q_{1}(0)=0.50, p(0)=0.00\right]$ when $\lambda=-0.0372$, and $N=0.3720$ : (a) the time history of the structural motion; (b) the phase portrait of the structural motion; (c) the time history of the van der Pol oscillation; (d) the phase portrait of the van der Pol oscillation.

bifurcation is another bifurcation type analyzed in this paper, which indicates the stability changing of the responses, resulting in the occurrences for the jump phenomenon and multiple solutions. A typical point $(\sigma=0.016)$ between the two saddle-node bifurcation points when $\lambda=0.0629$ and $N=0.2178$ in Region $I V$ as shown in Fig.8 is chosen to obtain two stable solutions by using the Runge-Kutta method. The other parameters are also fixed at $A=4.2 \times 10^{-4} \mathrm{~m}^{2}, \rho=7800 \mathrm{~kg} / \mathrm{m}^{3}, \rho_{f}=1.225 \mathrm{~kg} / \mathrm{m}^{3}, E I=300 \mathrm{~N} \cdot \mathrm{m}, V=100 \mathrm{~m} / \mathrm{s}$, $\Omega=450 \mathrm{rad} / \mathrm{s}, D_{0}=0.1 \mathrm{~m}, L=0.3 \mathrm{~m}, c=6 \mathrm{~N} \cdot \mathrm{s} / \mathrm{m}, \mathrm{St}=0.1, C_{L 0}=0.3$. The two set$\mathrm{s}$ of time histories and phase portraits for the structural and van der Pol motions are ob- 


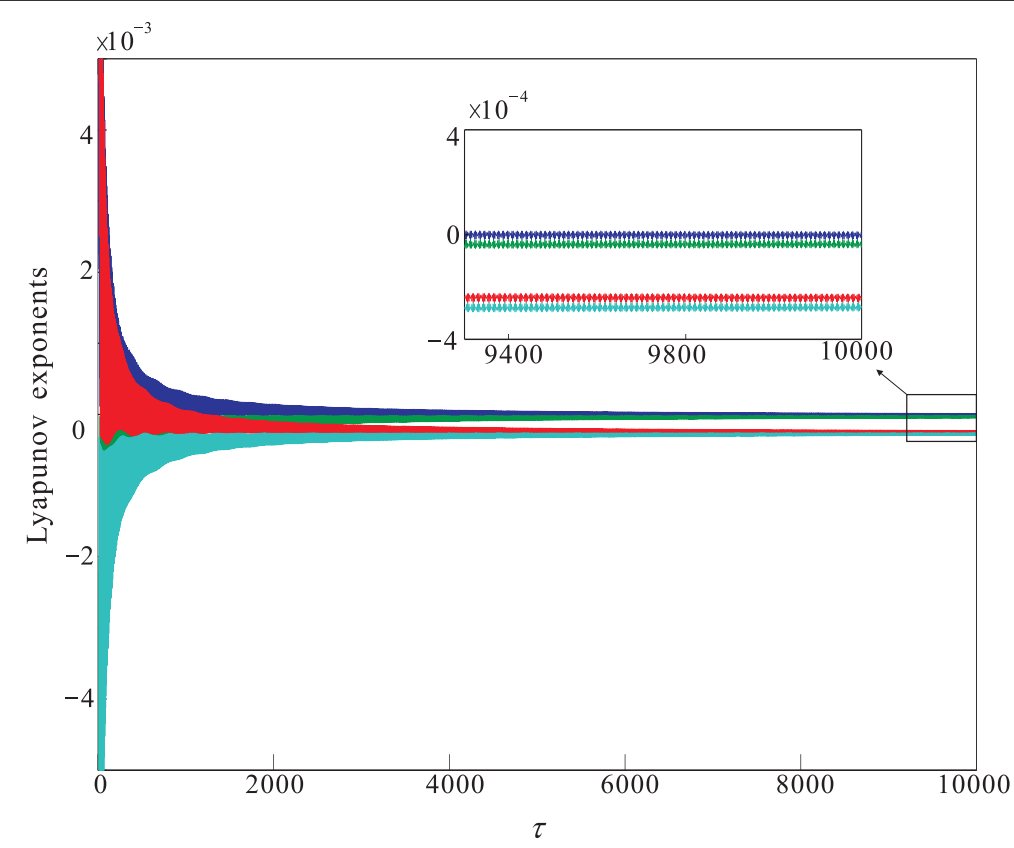

Fig. 18 The Lyapunov exponents for the original system at a typical point $(\sigma=0.0285)$ after the Hopf bifurcation as shown in Figure 5 with the initial conditions $\left[v_{1}(0)=\right.$ $\left.0.05, y(0)=0.00, q_{1}(0)=0.50, p(0)=0.00\right]$ when $\lambda=-0.0372$ and $N=0.3720$.

tained with the initial condition values $\left[v_{1}(0)=0.1, y(0)=0.0, q_{1}(0)=2.0, p(0)=0.0\right]$ and $\left[v_{1}(0)=0.40, y(0)=0.00, q_{1}(0)=2.65, p(0)=0.00\right]$, respectively, which are shown in Fig.19.

Moreover, the time histories of the two-degree-of-freedom motions can be predicted by Eqs. (23), (28), and (37)-(40) analytically. Comparisons of time histories obtained by the analytical and numerical methods for the two different solutions as shown in Fig.19 are carried out with the same system parameter values, which read $A=4.2 \times 10^{-4} \mathrm{~m}^{2}, \rho=7800 \mathrm{~kg} / \mathrm{m}^{3}, \rho_{f}=1.225 \mathrm{~kg} / \mathrm{m}^{3}$, $E I=300 \mathrm{~N} \cdot \mathrm{m}, V=100 \mathrm{~m} / \mathrm{s}, \Omega=450 \mathrm{rad} / \mathrm{s}, D_{0}=0.1 \mathrm{~m}, L=0.3 \mathrm{~m}, c=6 \mathrm{~N} \cdot \mathrm{s} / \mathrm{m}, \mathrm{St}=0.1$, $C_{L 0}=0.3$. Figs 20 and 21 show that the analytical results for time histories have a good agreement with the numerical ones obtained by the Runge-Kutta method.

\section{Conclusions}

The vortex-induced vibrations of a rotating blade were investigated, in which the blade was modeled as a uniform and straight cantilever beam while the van der Pol oscillator was used to simulate the time-varying of the vortex. The reaction for the motion of the blade on the fluid was represented by a linear inertial coupling. The multiple scale method was applied to analyze the 1:1 internal resonance of the coupled system. The bifurcation equations were derived and a two-parameter bifurcation diagram for the van der Pol damping $\lambda$ and the coupling parameter $N$ was obtained with the singularity theory of two state variables. The bifurcation characteristics for the frequency-responses in different bifurcation regions were investigated. The phenomena including the saddle-node and Hopf bifurcations were found to occur under certain parameter regions. The bifurcation analysis showed that when parameters $\lambda$ and $N$ have the same signs, namely $\lambda N>0$, the varying trends of the structural and van der Pol motions are similar to each other. Specifically, when both parameters $\lambda$ and $N$ are positive $(\lambda>0$ and $N>0$ ), two kinds of dynamic characteristics for the responses can occur: (a) the responses are totally stable as $\sigma$ varies (e.g. the responses in Region $V$ ); (b) the solutions can encounter the saddle-node bifurcation at a certain value for the detuning parameter $\sigma$ (e.g. the responses in 
(a)

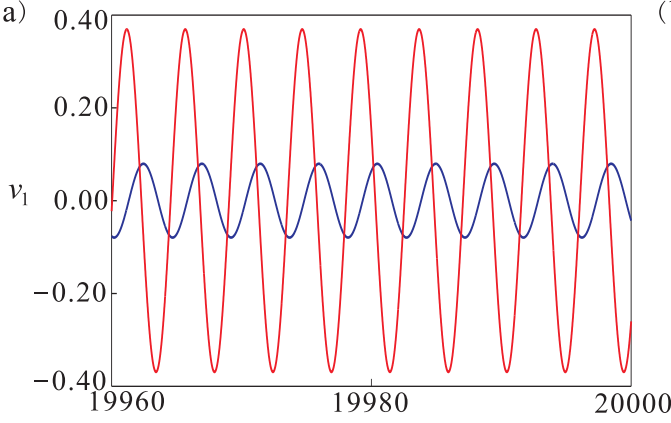

(c)

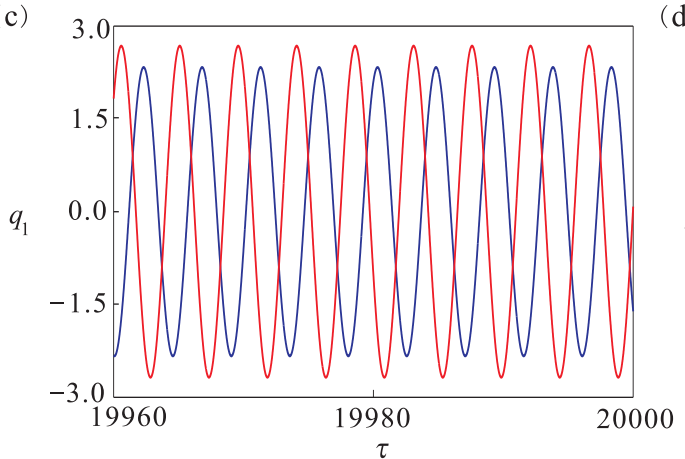

(b) 0.60

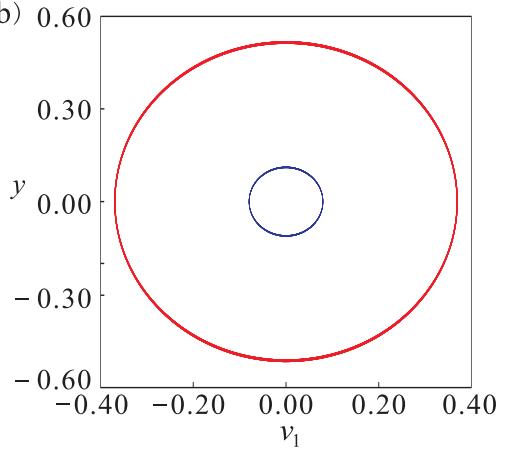

d)

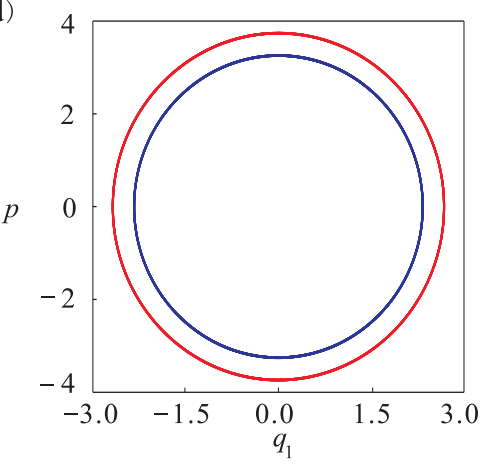

Fig. 19 The time histories and phase portraits of the multiple-solutions when $\lambda=0.0629, N=$ 0.2178 and $\sigma=0.0160$. The blue line indicates the vibrations with the initial condition values $\left[v_{1}(0)=0.1, y(0)=0.0, q_{1}(0)=2.0, p(0)=0.0\right]$, and the red line remarks the vibrations with the initial condition values $\left[v_{1}(0)=0.40, y(0)=0.00, q_{1}(0)=\right.$ $2.65, p(0)=0.00]$ : (a) the time histories of the structural motion; (b) the phase portraits of the structural motion; (c) the time histories of the van der Pol oscillation; (d) the phase portraits of the van der Pol oscillation.

Region $I V)$. When both values for parameters $\lambda$ and $N$ are negative $(\lambda<0$ and $N<0)$, the solutions are unstable ones as $\sigma$ varies (e.g. the responses in Region $V I I I$ ). While parameters $\lambda$ and $N$ have the opposite signs $(\lambda N<0)$, the varying trends of the two motions are opposite to each other, which means the energy transfer between the two modes. The Hopf bifurcation can be encountered for certain parameter values when parameters $\lambda$ and $N$ have the opposite signs. The time histories, phase portraits and Lyapunov exponents were computed with the Runge-Kutta method for the original system at a point after the Hopf bifurcation in Region $I I I$, results of which reveal that the occurrence of the Hopf bifurcation for the modulation equations indicating the quasi-periodic motions for the original system. Additionally, the coexisting multiple solutions generated for the saddle-node bifurcation in the parametric Region $I V$ have been illustrated, whilst their corresponding numerical and analytical time histories have been compared subsequently, both of which are in good agreement with each other. These results indicate the validity of the analytical solutions obtained by using the multiple scale method.

Acknowledgements The authors acknowledge the projects supported by the National Basic Research Program of China (973 Project)(No. 2015CB057405) and the National Natural Science Foundation of China (No. 11372082) and the State Scholarship Fund of CSC. DW thanks for the hospitality of the University of Aberdeen. 


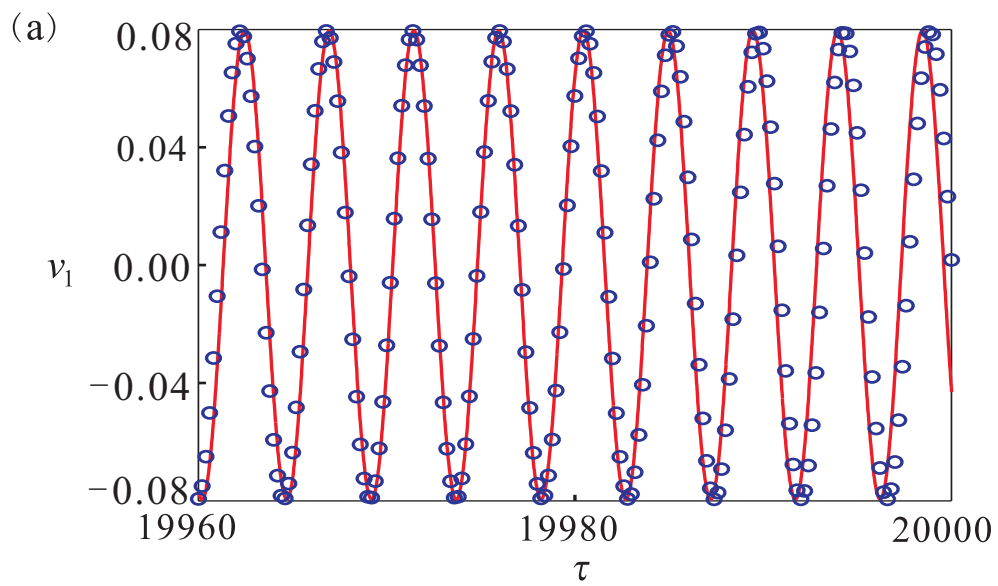

(b)

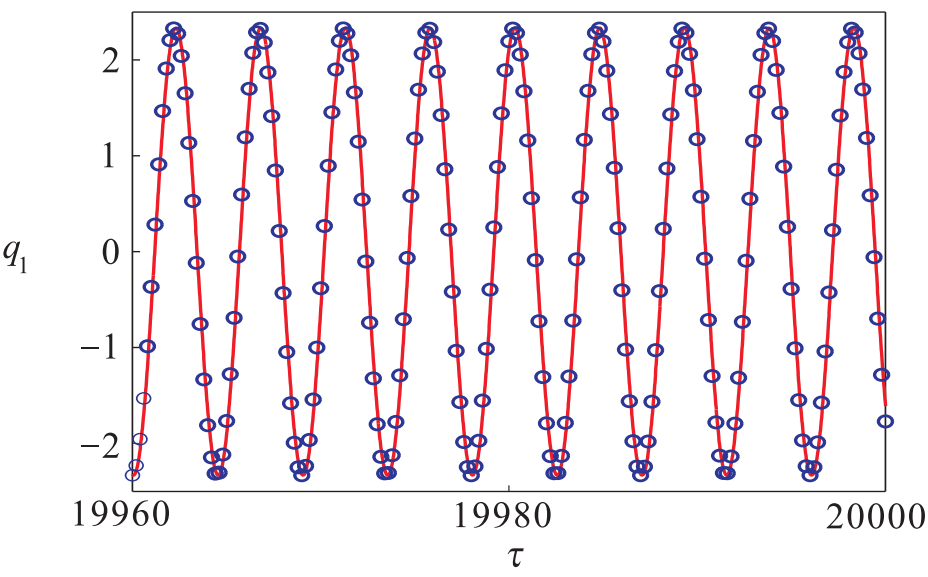

Fig. 20 Comparisons of the time histories obtained with the analytical and numerical methods when $\lambda=0.0629, N=0.2178$ and $\sigma=0.0160$ with the initial conditions $\left[v_{1}(0)=\right.$ $\left.0.1, y(0)=0.0, q_{1}(0)=2.0, p(0)=0.0\right]$ : (a) the time histories of the structural motion; (b) the time histories of the van der Pol oscillation. Solid lines and circled lines denote the analytical and numerical results, respectively

\section{References}

[1] C. H. K. Williamson, A. Roshko. Vortex formation in the wake of an oscillating cylinder. J. Fluid Struct. 2, 355-381 (1988)

[2] J. C. S. Lai , M. F.Platzer. Jet characteristics of a plunging airfoil. AIAA J. 37(12), 1529-1537 (1999)

[3] W. Shyy, M. Berg, D. Ljungqvist. Flapping and flexible wings for biological and micro air vehicles. Prog. Aerosp. Sci. 35, 455-505 (1999)

[4] J. P. Gostelow, M. F. Platzer, W. E. Carscallen. On vortex formation in the wake flows of transonic turbine blades and oscillating airfoils. J. Turbomach.-ASME, 128 528-535 (2006)

[5] O. Lawaczek, H. J. Heinemann. Von Kármán vortex streets in the wakes of subsonic and transonic cascades. AGARD CP-177. (1976)

[6] C. H. Sieverding, H. Heinemann. The influence of boundary layer state on vortex shedding from flat plates and turbinecascades. ASME Paper No. 89-GT-296. (1989)

[7] P. Beauseroy, R. Lengelle. Nonintrusive turbomachine blade vibration measurement system. Mech. Syst. Signal Pr. 21, 1717-1738 (2007) 
(a)

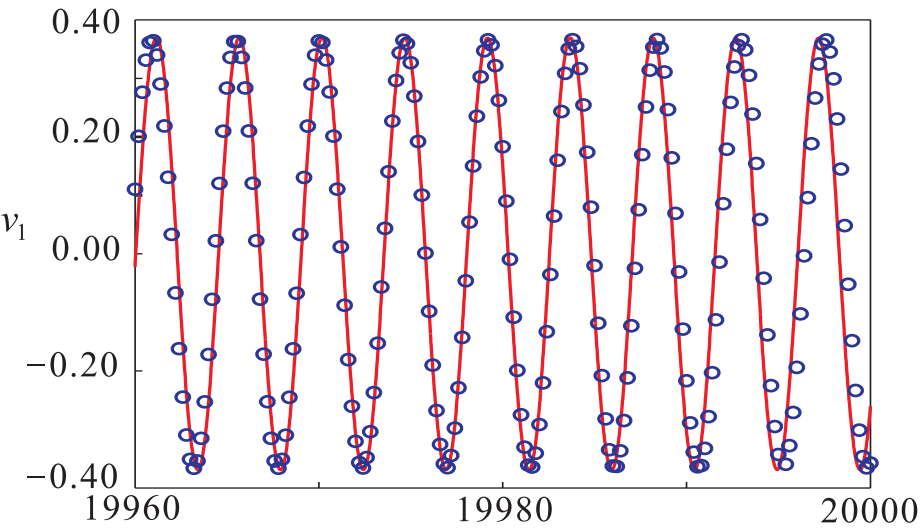

(b)

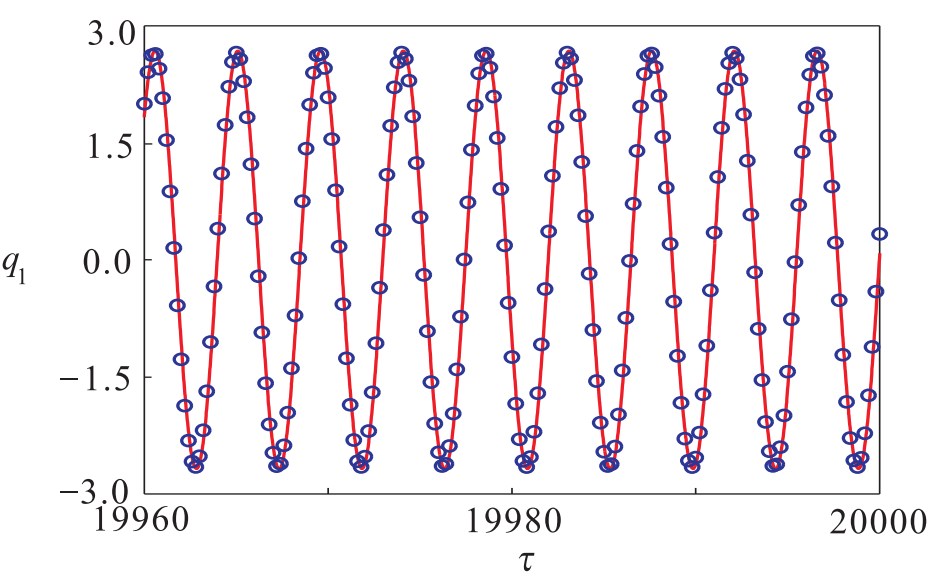

Fig. 21 Comparisons of the time histories obtained with the analytical and numerical method when $\lambda=0.0629, N=0.2178$ and $\sigma=0.0160$ with the initial conditions $\left[v_{1}(0)=\right.$ $\left.0.40, y(0)=0.00, q_{1}(0)=2.65, p(0)=0.00\right]$ : (a) the time histories of the structural motion; (b) the time histories of the van der Pol oscillation. Solid lines and circled lines denote the analytical and numerical results, respectively

[8] C. G. Rodriguez, E. Egusquiza, I.F. Santos. Frequencies in the vibration induced by the rotor stator interaction in a centrifugal pump turbine. J. Fluid Eng-T ASME 129, 1428-1435 (2007)

[9] R. Violette, E. de Langre, J. Szydlowsky. Computation of vortex-induced vibrations of long structures using a wake oscillator model: Comparison with DNS and experiments. Comput. Struct. 85, 1134-1141 (2007)

[10] K. B. Skaugset, C. M. Larsen. Direct numerical simulation and experimental investigation on suppression of vortex induced vibrations of circular cylinders by radial water jets. Flow Turbul. Combust. 71, 35-59 (2003)

[11] E. Guilmineau, P. Queutey. Numerical simulation of vortex-induced vibration of a circular cylinder with low mass-damping in a turbulent flow. J. Fluid Struct. 19, 449-466 (2004)

[12] J. S. Rao, A. Saldanha. Turbomachine blade damping. J. Sound Vib. 262, 731-738 (2003)

[13] G. Dimitriadis, I. B. Carrington, J. R. Wright, J. E. Copper. Blade-tip timming measurement of synchronous vibrations of rotating bladed assemblies. Mech. Syst. Signal Pr. 16, 599-622 (2002)

[14] S. Kumar, N. Roy, R. Ganguli. Monitoring low cycle fatigue damage in turbine blade using vibration characteristics. Mech. Syst. Signal Pr. 21, 480-501 (2007)

[15] M. A. Barron, M. Sen. Synchronization of coupled self-excited elastic beams. J. Sound Vib. 324, 209-220 (2009) 
[16] M. A. Barron. Vibration analysis of a self excited elastic beam. J. Appl. Res. Technol. 8(2), 227-239 (2010)

[17] D. Cao, X. Gong, D. Wei, et al. Nonlinear vibration characteristics of a flexible blade with friction damping due to tip-rub. Shock Vib. 18, 105-114 (2011)

[18] S. Chu, D. Cao, S. Sun, et al. Impact vibration characteristics of a shrouded blade with asymmetric gaps under wake flow excitations, Nonlinear Dyn. 72, 539-554 (2013)

[19] R. E. D. Bishop, A. Y. Hassan. The lift and drag forces on a circled cylinder in a flowing fluid. $P$. Roy. Soc. A-Math. Phy. 277(1368), 32-50 (1964)

[20] P. Hemon. An improvement of the time delayed quasi-steady model for the oscillations of circular cylinders in cross-flow. J. Fluid Struct. 13, 291-307 (1999)

[21] R. Gabbai, H. Benaroya. An overview of modeling and experiments of vortex-induced vibration of circular cylinders, J. Sound Vib. 282, 575-616 (2005)

[22] Y. Lee, A. Vakakis, L. Bergman, M. McFarland. Suppression of limit cycle oscillations in the van der Pol oscillator by means of passive nonlinear energy sinks. Struct. Contr. Health Monit. 13, 41-75 (2006)

[23] R. Hartlen, I. Currie. Lift-oscillator model of vortex induced vibration. J. Eng. Mech.-ASCE 96, 577-591 (1970)

[24] R. Skop, O. Griffin. A model for the vortex-excited resonant response of bluff cylinders. J. Sound Vib. 27, 225-233 (1973)

[25] M. L. Facchinetti, E. de Langre, F. Biolley. Coupling of structure and wake oscillators in vortexinduced vibrations. J. Fluid Struct. 19, 123-140 (2004)

[26] M. Keber, M. Wiercigroch. A reduced order model for vortex-induced vibration of a vertical offshore riser in lock-in. IUTAM symposium on fluid-structure interaction in Ocean engineering, Iutam Bookseries Volume 8, 155-166 (2008)

[27] D. Wang, Y. Chen, M. Wiercigroch et al. A three-degree-of-freedom model for vortex-induced vibrations of turbine blades. Meccanica Doi: 10.1007/s11012-016-0381-7, 1-22 (2016)

[28] D. Wang, Y. Chen, Z. Hao et al. Bifurcation analysis for vibrations of a turbine blade excited by air flows. Sci. China Tech. Sci. 59(6), 1-15 (2016)

[29] C. H. K. Williamson, R. Govardhan. A brief review of recent results in vortex-induced vibrations. J. Wind Eng. Ind. Aerod. 96, 713-735 (2008)

[30] R. A. Kadlec, S. S. Davis. Visualization of quasiperiodic flows. AIAA J. 17(11), 1164-1169 (1996)

[31] H. Ohashi, N. Ishikawa. Visualization study of a flow near the trailing edge of an oscillating airfoil. Bull. JSME 15, 840-845 (1972)

[32] M. M. Koochesfahani. Vortical patterns in the wake of an oscillating airfoil. AIAA J. 27(9), 1200-1205 (1989)

[33] J. Young, J. C. S. Lai. Oscillation frequency and amplitude effects on the wake of a plunging airfoil. AIAA J. 42(10), 2042-2052 (2004)

[34] E. Pesheck, C. Pierre, S.W. Shaw. Modal reduction of a nonlinear rotating beam through normal modes. J. Vib. Acoust.-ASME 124, 229-236 (2002)

[35] Özgür Turhan, Gökhan Bulut. On nonlinear vibrations of a rotating beam. J. Sound Vib. 322, 314-335 (2009)

[36] Z. Xu, X. Li, J. P. Park, S. J. Ryu. Effecr of Coriolis acceleration on dynamic characteristics of high speed spinning steam turbine blades. Journal of Xi'an Jiaotong University, 37(9): 894-897 (2003)

[37] R. W. Clough, J. Penzien. Dynamics of structures. Computers and Structures, Inc., 3rd edition, (2003)

[38] R. A. Skop, S. Balasubramanian. A new twist on an old model for vortex-excited vibration. $J$. Fluid Struct. 11, 395-412 (1997)

[39] N. Srinil, M. Wiercigroch, P. O'Brien. Reduced-order modelling of vortex-induced vibration of catenary riser, Ocean Eng. 36, 1404-1414 (2009)

[40] H. Xue, W. Tang, S. Zhang, Simplified model for evaluation of VIV- induced fatigue damage of deepwater marine risers. Journal of the Shanghai University (Sci.), 14(4), 435-442(2009) 
[41] M. Lucca Facchinetti, E. de Langre, F. Biolley. Vortex-induced travelling waves along a cable. Eur. J. Mech. B-Fluid, 23, 199-208 (2004)

[42] M. L. Facchinetti, E. de Langre, F. Biolley. Vortex shedding modeling using diffusive van der Pol oscillators. C. R. Mecanique, 330, 451-456 (2002)

[43] M. Keber. Vortex-Induced vibration of offshore risers: Theoretical modelling and analysis. A PhD thesis, University of Aberdeen. (2012)

[44] Z. Hao, Q. Cao. The isolation characteristics of an archetypal dynamical model with stable-quasizero-stiffness. J. Sound Vib., 340, $61-79$ (2015)

[45] Z. Hao, Q. Cao, M. Wiercigroch. Two-sided damping constraint control for high-performance vibration isolation and end-stop impact protection. Nonlinear Dyn., Doi:10.1007/s11071-016-26855, 1-16 (2016)

[46] A. H. Nayfeh, D. T. Mook. Nonlinear oscillations. New York, 331-338 (1979)

[47] Y. Wang, F. Li. Nonlinear primary resonance of nano beam with axial initial load by nonlocal continuum theory. Int. J. Nonlin. Mech. 61, 74-79 (2014)

[48] Q. Bi, Y. Chen. Bifurcation analysis of a double pendulum with internal resonance. Appl. Math. Mech.-Engl. Ed., 21(3), 255-264 (2000)

[49] Y. Chen, A. Y. T. Leung. Bifurcation and chaos in engineering, Springer-Verlag, London (1998)

[50] M. Golubitsky, D. G. Schaeffer. Singluarities and groups in bifurcation theory-I, Springer-Verlag, New York (1984)

[51] X. Wang, Y. Chen, L. Hou. Nonlinear dynamic singularity analysis of two interconnected synchronous generator system with 1:3 internal resonance and parametric principal resonance. Appl. Math. Mech.-Engl. Ed., 36(8), 985-1004 (2015)

[52] Z. Qin, Y. Chen, J. Li. Singularity analysis of a two-dimensional elastic cable with 1:1 internal resonance. Appl. Math. Mech.-Engl. Ed., 31(2), 143-150 (2010)

[53] G. Schmidt, A. Tondl. Nonlinear vibration. Cambrige: Cambrige University Press. (1986)

[54] M. Monteil, C. Touzé, O. Thomas, S. Benacchio. Nonlinear forced vibrations of thin structures with tuned eigenfrequencies: the cases of 1:2:4 and 1:2:2 internal resonances. Nonlinear Dyn. 75, 175-200 (2014)

Appendix A The partial derivatives of the bifurcation functions with respect to $\sigma, a_{1}$ and $a_{2}$ are derived as shown in the following:

$$
\begin{aligned}
& G_{1 a_{1}}=-2 \omega_{s}^{3} N \zeta a_{1}, \\
& G_{1 a_{2}}=\frac{1}{4} \lambda \omega_{R}^{2}\left(b \omega_{R}^{2}+d\right)\left(3 a_{2}^{2}-8\right) a_{2}, \\
& G_{1 \sigma}=0, \\
& G_{2 a_{1}}=-8 \omega_{s}^{2} \omega_{R}^{2} \sigma^{2} a_{1} a_{2}^{2}-2 \omega_{s}^{2} \omega_{R}^{2} a_{1} a_{2}^{2}\left[\zeta+\left(\frac{3 a_{2}^{2}}{16}-1\right) \lambda \omega_{R}\right]^{2}+ \\
& 4 \omega_{s}^{3} N a_{1}\left[\omega_{R}\left(b \omega_{R}^{2}+d\right) a_{2}^{2}+N \omega_{s}^{3} a_{1}^{2}\right], \\
& G_{2 a_{2}}=-8 \omega_{R}^{2} \omega_{s}^{2} \sigma^{2} a_{1}^{2} a_{2}-\frac{3}{4} \lambda \omega_{R}^{3} \omega_{s}^{2} a_{1}^{2} a_{2}^{3}\left[\zeta+\left(\frac{3 a_{2}^{2}}{16}-1\right) \lambda \omega_{R}\right]- \\
& 2 \omega_{R}^{2} \omega_{s}^{2} a_{1}^{2} a_{2}\left[\zeta+\left(\frac{3 a_{2}^{2}}{16}-1\right) \lambda \omega_{R}\right]^{2}+4 \omega_{R} a_{2}\left(b \omega_{R}^{2}+d\right)\left[\omega_{R}\left(b \omega_{R}^{2}+d\right) a_{2}^{2}+N \omega_{s}^{3} a_{1}^{2}\right] \\
& G_{2 \sigma}=-8 \omega_{R}^{2} \omega_{s}^{2} \sigma a_{1}^{2} a_{2}^{2}, \\
& G_{1 a_{1}, a_{1}}=-2 N \omega_{s}^{3} \zeta \text {, } \\
& G_{1 a_{1}, a_{2}}=0 \text {, } \\
& G_{1 a_{2}, a_{2}}=\frac{1}{4} \lambda \omega_{R}^{2}\left(b \omega_{R}^{2}+d\right)\left(9 a_{2}^{2}-8\right), \\
& G_{2 a_{1}, a_{1}}=-8 \sigma \omega_{R}^{2} \omega_{s}^{2} a_{2}^{2}-2 \omega_{s}^{2} \omega_{R}^{2} a_{2}^{2}\left[\zeta+\left(\frac{3 a_{2}^{2}}{16}-1\right) \lambda \omega_{R}\right]^{2}+ \\
& 8 N^{2} \omega_{s}^{6} a_{1}^{2}+4 N \omega_{s}^{3}\left[\omega_{R} a_{2}^{2}\left(b \omega_{R}^{2}+d\right)+N \omega_{s}^{3} a_{1}^{2}\right],
\end{aligned}
$$




$$
\begin{gathered}
G_{2 a_{2}, a_{1}}=G_{2 a_{1}, a_{2}}=-16 \sigma \omega_{R}^{2} \omega_{s}^{2} a_{1} a_{2}-\frac{3}{2} \lambda \omega_{s}^{2} \omega_{R}^{3} a_{1} a_{2}^{3}\left[\zeta+\left(\frac{3 a_{2}^{2}}{16}-1\right) \lambda \omega_{R}\right]- \\
4 \omega_{R}^{2} \omega_{s}^{2} a_{1} a_{2}\left[\zeta+\left(\frac{3 a_{2}^{2}}{16}-1\right) \lambda \omega_{R}\right]^{2}+8 \omega_{R} \omega_{s}^{3} N\left(b \omega_{R}^{2}+d\right) a_{1} a_{2} \\
G_{2 a_{2}, a_{2}}=-\frac{135}{128} \lambda^{2} \omega_{R}^{4} \omega_{s}^{2} a_{1}^{2} a_{2}^{4}-2 \omega_{R} \omega_{s}^{2} a_{1}^{2}\left(\omega_{R} \zeta^{2}+4 \omega_{R} \sigma-2 \zeta \lambda \omega_{R}^{2}+\lambda^{2} \omega_{R}^{3}-2 d N \omega_{s}-2 b N \omega_{R}^{2} \omega_{s}\right) \\
+\frac{3}{2} \omega_{R}^{2} a_{2}^{2}\left\{8 d^{2}+16 \omega_{R}^{2} b d+\omega_{R}\left[8 b^{2} \omega_{R}^{3}+3 \omega_{s}^{2} \lambda\left(\lambda \omega_{R}-\zeta\right) a_{1}^{2}\right]\right\}
\end{gathered}
$$

